\title{
Vortex Trapping Cavity on Airfoil: High-Order Penalized Vortex Method Numerical Simulation and Water Tunnel Experimental Investigation
}

\author{
Dominik Błoński *(D), Katarzyna Strzelecka * and Henryk Kudela (D) \\ Faculty of Power and Mechanical Engineering, Wrocław University of Science and Technology, \\ Wybrzeże Wyspiańskiego 27, 50-370 Wrocław, Poland; henryk.kudela@pwr.edu.pl \\ * Correspondence: dominik.blonski@pwr.edu.pl (D.B.); katarzyna.strzelecka@pwr.edu.pl (K.S.)
}

check for updates

Citation: Błoński, D.; Strzelecka, K.; Kudela, H. Vortex Trapping Cavity on Airfoil: High-Order Penalized Vortex Method Numerical Simulation and Water Tunnel Experimental Investigation. Energies 2021, 14, 8402. https://doi.org/10.3390/en14248402

Academic Editor: John Ringwood

Received: 30 September 2021

Accepted: 29 November 2021

Published: 13 December 2021

Publisher's Note: MDPI stays neutral with regard to jurisdictional claims in published maps and institutional affiliations.

Copyright: (c) 2021 by the authors. Licensee MDPI, Basel, Switzerland. This article is an open access article distributed under the terms and conditions of the Creative Commons Attribution (CC BY) license (https:/ / creativecommons.org/licenses/by/ $4.0 /)$.

\begin{abstract}
This paper presents a two-dimensional implementation of the high-order penalized vortex in cell method applied to solve the flow past an airfoil with a vortex trapping cavity operating under moderate Reynolds number. The purpose of this article is to investigate the fundamentals of the vortex trapping cavity. The first part of the paper treats with the numerical implementation of the method and high-order schemes incorporated into the algorithm. Poisson, stream-velocity, advection, and diffusion equations were solved. The derivation, finite difference formulation, Lagrangian particle remeshing procedure, and accuracy tests were shown. Flow past complex geometries was possible through the penalization method. A procedure description for preparing geometry data was included. The entire methodology was tested with flow past impulsively started cylinder for three Reynolds numbers: 550, 3000, 9500. Drag coefficient, streamlines, and vorticity contours were checked against results obtained by other authors. Afterwards, simulations and experimental results are presented for a standard airfoil and those equipped with a trapping vortex cavity. Airfoil with an optimized cavity shape was tested under three angles of attack: $3^{\circ}, 6^{\circ}, 9^{\circ}$. The Reynolds number is equal to $R e=2 \times 10^{4}$. Apart from performing flow analysis, drag and lift coefficients for different shapes were measured to assess the effect of vortex trapping cavity on aerodynamic performance. Flow patterns were compared against ultraviolet dye visualizations obtained from the water tunnel experiment.
\end{abstract}

Keywords: penalized vortex particle; trapping vortex cavity; airfoil numerical simulation; finite difference

\section{Introduction}

In the era of growing demand for electricity production from renewable energy sources, research and development work on improving the efficiency of fluid flow machines are particularly important. Wind turbines as well as hydro turbines are some of the most important devices used for the generation of renewable energy. Due to the widespread usage of these machines, the maximization of their efficiency is desired. A well-designed blade system is a crucial element of these turbines and improving their aerodynamic performance will have a significant impact on the overall turbine performance. There are various high-thickness symmetrical (e.g., NACA0024 [1], NACA0018 [2,3]) as well as asymmetrical (e.g., Risø [4]) airfoils which are used in both vertical and horizontal wind turbines and hydro turbines.

The reduction and control of flow separation are some of the main points of interest for designers working to improve airfoil performance. The kinetic energy loss is dependent on the separation area size and causes undesirable effects. It contributes to the increase of the drag force. The lift force loss at high angles of attack is also closely related to flow separation on the upper (suction) surface of the airfoil. It is therefore reasonable to say that moving this separation towards the trailing edge is key for airfoil performance [5]. 
A delayed flow separation results in the postponement of the stall during the turbine blade's work at a high angle of attack. What is more, it can be assumed that the reduction of the recirculating flow can minimize the wake region, and hence the drag force decreases. That is crucial for the power coefficient that strongly depends on the lift-to-drag coefficient ratio $\left(C_{L} / C_{D}\right)$ [6]. A modern airfoil can have a lift force two hundred times bigger than the drag force. With the increase of its value, the peak of the power coefficient shifts towards a higher tip speed ratio (TSR). The tip speed ratio is a parameter that describes the work regime of a machine. It is defined as a multiplication of the angular velocity and the radius of the rotor divided by the free stream velocity. In addition, an increase of the $C_{L} / C_{D}$ ratio and operation at a higher TSR decreases the impact of the number of rotor blades on the maximum power coefficient. In other words, a low-speed rotor requires a higher amount of blades but their shapes do not have a significant impact on the power output. High TSR rotors will perform well with a smaller amount of blades, but the airfoil lift-to-drag ratio value is decisive when it comes to maximizing the power coefficient.

Apart from airfoil geometry, the $C_{L} / C_{D}$ ratio is strongly influenced by flow conditions, namely - the Reynolds number. As the Reynolds number increases, the thickness of the boundary layer decreases, which reduces the drag force. The value of the lift force coefficient remains constant, or, it changes by a small amount [7].

When the critical angle of attack is exceeded, an increase in the separated flow area is caused, and thus a rapid loss of the lift force and an increase in the drag force, and consequently, the $C_{L} / C_{D}$ ratio decreases. There are various methods for changing the critical angle of attack. They can be categorized as passive or active methods [8-15] depending on whether they require the usage of additional power or a steering system. Using both methods yields transition delay which leads to increased lift and reduced drag force. The use of a maintenance-free method that could postpone separation without any other disturbance in the flow at a low cost would be most beneficial.

A trapped vortex cavity (TVC) is one of the methods that can be used as a passive method at a low cost. The potential advantage of using TVC is that the presence of the cavity on the wing surface naturally causes the vortex-formation inducing flow reattachment that requires no auxiliary power. Therefore, the separated flow is forced to remain attached by an intense vortex, anchored in the cavity. One of the critical points to consider is maintaining the vortex stability in the cavity, and with sufficient strength to force the flow reattachment. The principle of the method is that the generated (trapped) vortex reduces flow separation downstream of the cavity, leading to a smaller area of the wake region [16-18], stabilized vortices, and an increased lift-to-drag ratio [16].

Numerous researchers have investigated the use of a cavity on a wall and airfoils [19-22]. Recent researches focus on shape optimization and the testing of cavity effectiveness located on the suction side of the airfoil.

Olsman and Colonius [17] presented a two-dimensional numerical simulation of the flow with a low Reynolds number $(R e)$ over a NACA0018 airfoil with a cavity. The global effect of the cavity on the flow around the airfoil was the generation of vortices that reduced flow separation downstream of the cavity. At high positive angles of attack (e.g., $\alpha=10^{\circ}$ ) the flow was separated well before the forward edge of the cavity and the cavity was in the separation bubble. Then the separated flow interacted with the cavity causing the generation of smaller-scale eddies and a narrower wake compared with the basis airfoil. The authors suggested a higher lift-to-drag ratio for the airfoil with a cavity compared to the airfoil without a cavity.

Olsman et al. [23] focused on both steady flow and a vertical translational motion at a low amplitude of the NACA 0018 airfoil, simulated in a wind tunnel via acoustic forcing. They investigated the unsteady behavior of the flow past airfoils with and without cavities using local pressure measurements, flow visualization, and hot-wire anemometry.

Yeung [24] tested the possibility of trapping single and multiple vortices on an airfoil. The results showed that an indented surface can stabilize a trapped vortex. The study 
also indicated that multiple vortex trapping is possible, although the chaotic motion of the vortices was found.

Some results are also available for the symmetric airfoil, with the cavity located near the leading edge (LE) or the trailing edge (TE) [16-18,25]. Vuddagiri and Samad (2013) [26] presented the flow over an airfoil with different cavity shapes placed on the suction surface of a symmetric airfoil both-near the LE and TE. Lift and drag were checked. The location of the separation point was identified at different Reynolds numbers $(R e)$ and different angles of attack for the airfoil with and without a cavity. The improvement in the critical angle of attack was observed for the cavity placed near the TE though the lift coefficient had a lower value as compared to the airfoil without a cavity. The cavity with sharp edges resulted in higher lift and lower drag as compared to the cavity having chamfered edges.

Vuddagiri et al. (2016) [27] investigated the effect of a circular cavity in various locations on an airfoil. They showed that the cavity placed on the trailing edge produced more lift compared with all of the other analyzed locations.

Some authors have tested various shapes for cavity optimization. Fatehi et al. (2019) [28] showed that the numerically optimized cavity on the suction surface significantly increased the lift-to-drag ratio. Nili-Ahmadabadi et al. (2020) [29] optimized a cavity on a Risø-B1-18 airfoil at an off-design angle of attack. For the optimization, the authors defined the cavity geometry (the wall shape, depth, and mouth of the cavity, as well as the height of the cavity), location of the cavity, and downstream upper surface by 16 parameters. Two models of Risø-B1-18 airfoils with and without the cavity were tested in an open-jet wind tunnel. It was shown in the PIV research that the optimized cavity trapped a vortex, which postponed the stall angle by about $3^{\circ}$. Starting from the angle of attack $\alpha=9^{\circ}$, the drag coefficient for the profile with the cavity was lower than for the standard profile.

In some works on trapping vortex cavities, the authors point out that a stable flow with trapped vortices rarely occurs. Therefore, it is proposed to additionally use active flow control inside the cavity to stabilize the vortices [16,30]. Gregorio and Fraioli [16] investigated the potential benefit obtainable by using a trapping vortex cell (TVC) system on a high thickness airfoil with and without steady suction. The authors found that a passive TVC was not able to control the flow separation. The vortex was not confined in a cavity and vortex shedding was observed. This reduced the aerodynamic properties of the airfoil compared to the original profile. Active TVC flow control was able to control the flow separation and full flow reattachment was achieved under certain conditions.

Lasagna et al. [31] experimentally tested the effectiveness of a trapped vortex cell in controlling the flow past a thick wing profile (NACA0024) at $R e=10^{6}$ and $6.67 \times 10^{5}$. The static pressure distributions around the model and the wake velocity profiles were measured to obtain lift and drag coefficients, for a standard airfoil and the airfoil with trapped vortex cell (TVC) configurations-with and without suction. Suction was applied in the cavity region to stabilize the trapped vortex. For comparison, a classical boundary layer suction configuration was also tested. A strong influence of the angle of attack, the suction rate, and the Reynolds number on the drag coefficient were observed. For the standard NACA0024 airfoil, the control to a drag reduction occurred only if the suction was high enough. Compared to the classical boundary layer suction configuration, the drag reduction was higher for the same amount of suction only for the angle of attack in the range of $\alpha=-2^{\circ}$ to $\alpha=6^{\circ}$ for $R e=10^{6}$. For other conditions, the classical boundary layer suction configuration was found to give better drag performances.

Utilization of vortex trapping cavity effect can be advantageous when designing new turbomachinery flow-paths. A deep understanding of the phenomena occurring under the flow around an airfoil with a cavity is a substantial step in the improvement of blade system performance. In this paper, the authors try to present the impact of a vortex trapping cavity on airfoil performance. Since we are dealing with unsteady behavior of highly vortical flow, utilizing the vortex particle method is the most suitable approach.

The vortex particle methods belong to the Lagrangian methods and they are preferred for advection-dominated flows. They are well-established and efficient with a history 
reaching back to the 1970s. Through the years, it was successfully used to perform numerical simulations of various physical phenomena. The fundamentals of vortex particle methods are given in [32]. A complete review of recent advances in vortex methods and their applications was presented by Mimeau [33].

Vortex particle methods are known for their ability to parallelize computations. The presented parallel computations for the 3D flow using the graphics card (GPU) are presented in [34]. It is also worth mentioning the work of van Rees et al. [35], in which implementation adapted to obtain high accuracy of vortex method, and was compared against spectral methods. Recent works in the field of particle methods focus on dealing with high Reynolds number flows, which include vortex large eddy simulation [36] (LES) and adaptive mesh refinement [37] (AMR).

Among the vortex particle methods, we can distinguish the vortex in cell method which is known to be a hybrid method. In this method, advection is solved in the Lagrangian framework, while the diffusion is solved on the Eulerian grid. This method has been proven to simulate flows with the existence of solid bodies, [38]. To fit the grid to the solid body boundary, conformal mapping can be used. However, this can be troublesome when dealing with complex and multiple geometries.

The big advantage of the technique used in this paper is the ability to perform calculations for any shape while maintaining a simple and accurate algorithm. A comprehensive study on the justification of high-order compact formulation for linear solvers application was done in $[39,40]$.

In this work, the greatest contribution to the field of computational fluid mechanics is to propose a method of penalized vortex in cell method suitable for any geometry based on the imported CAD files. This method was tested for the flow over the cylinder and was applied for simulating the flow past an airfoil with a vortex trapping cavity.

\section{Equation of Fluid Motion and Penalized Vortex in Cell Method}

The possibility of analyzing the fluid flow phenomena in terms of vorticity dynamics is still very attractive and desirable. It is convenient to determine fluid motion in terms of vorticity. Mass and momentum conservation equations for two-dimensional, incompressible, and laminar Newtonian flow in primitive variables formulation are:

$$
\begin{gathered}
\frac{\partial \mathbf{u}}{\partial t}+(\mathbf{u} \cdot \nabla) \mathbf{u}-v \nabla^{2} \mathbf{u}+\frac{1}{\rho} \nabla p=0 \\
\nabla \mathbf{u}=0
\end{gathered}
$$

where $\mathbf{u}$ us the velocity vector, $v$ is the kinematic viscosity, $\rho$ is density, and $p$ is the pressure.

A flow in which a rigid body is present can be described as a flow through a porous zone with varying permeability. Fluid is a high permeability medium and a solid body is a low permeability medium. Such flow is described with Navier-Stokes-Brinkman equations for incompressible viscous flow. According to [41] such fluid motion is described as:

$$
\frac{\partial \mathbf{u}}{\partial t}+(\mathbf{u} \cdot \nabla) \mathbf{u}-v \nabla^{2} \mathbf{u}+\frac{1}{\rho} \nabla p=\lambda \chi \mathbf{u}
$$

This equation can be transformed to the Helmholtz equations that govern the evolution of the vorticity:

$$
\begin{gathered}
\frac{\partial \omega}{\partial t}+(\nabla \omega) \cdot \mathbf{u}=v \Delta \omega+\Delta \times(\chi \lambda \mathbf{u}) \\
\Delta \psi=-\omega
\end{gathered}
$$

For the solution of Equation (4), the vortex particle method is the preferred approach when it comes to simplicity and computational efficiency. It is highly recommended when dealing with studies of oscillatory motions that create great flow separation around bodies. The equation is solved using the viscous splitting algorithm: at first, the non-viscous part 
(advection) of Equation (4) is solved by the Lagrangian particle method and in the second step the viscosity of the fluid is taken into account (diffusion equation). The presence of a rigid body is taken into account by the penalization method [42,43]. A continuous vorticity field is approximated by the system of the vortex particles. Due to hardware limitations, the number of particles is limited. A single particle corresponds to the mean vorticity of one cell with an area equal to $S_{p}=h^{2}$. Each particle has circulation assigned according to the equation:

$$
\Gamma_{p}=\int_{S_{p}} \omega d S .
$$

Vortex particles are placed on the grid nodes and are advected in the velocity field of the fluid. The grid was used for solving Poisson Equation $(5)(\psi, \omega)$, velocity-stream function, and for solving the viscous step-diffusion equation. The Poisson equation was used to calculate the stream function needed to calculate velocity:

$$
\mathbf{u}=(u, v)=\left(\frac{\partial \psi}{\partial y},-\frac{\partial \psi}{\partial x}\right)
$$

Since vorticity is transported as a part of the inviscid step, relying on the Helmholtz and Kelvin circulation theorem, its value does not change with time. Therefore, vortex particles can be treated as material particles. Their displacement can be described by an ordinary differential equation:

$$
\frac{d \mathbf{x}_{\mathbf{p}}}{d t}=\mathbf{u}_{\mathbf{p}}
$$

where $\mathbf{u}_{\mathbf{p}}$ is known particle velocity. The no-slip boundary condition was obtained by penalization of the velocity field using the Brinkman penalization method. The impact on the velocity field and vorticity generation connected with the presence of a solid body is calculated with two steps. The first one corrects the velocity field based on the Brinkman equation. The second step corrects the vorticity field from the corrected velocity field. After the displacement of the particles, redistribution of the mass (circulation) back to the grid nodes is accomplished. The process of redistribution of the particle mass was done by a $\mathrm{Z}_{2}$ cardinal splines interpolation kernel, that conserves the moment of vortex particle up to the 2nd degree (zero-quantity of vorticity, first-impulse, second-angular impulse). Near the boundary, one-side redistribution functions were implemented. Because of the large grid size and number of time steps, all computation was done in parallel with the MPI. The systems of linear equations were solved with the hypre library [44].

The third section of the paper covers the process of achieving a highly accurate, fourthorder finite difference solver. The Poisson equation was solved using a compact, 9-point stencil. The computation of the velocity was achieved by using a 3-point compact scheme, which utilized Hermite interpolation. Advection of vortex particles was done by the fourth-order Runge-Kutta method with 4 nearest point velocity linear interpolation. The diffusion equation was solved using a factorization-based method connected with Hermite interpolation, Crank-Nicholson scheme. The main objective of the implemented algorithm was to keep the method compact, meaning that in the finite-difference formulation only the adjacent grid points are used for calculations. The global order of accuracy with respect to space is $O\left(h^{2}\right)$. Accuracy in respect to time is $O(\Delta t)$. Orders of accuracy are directly related to the penalization and viscous splitting algorithm.

The second part of the article discusses the numerical simulation and dye visualization of flow past an airfoil with a vortex trapping cavity. Results have been presented for various cavity geometries and angles of attack. In the article, an enhanced accuracy compact vortex in a cell implementation used for the simulation of flow past an airfoil was presented. The authors focused on an optimized cavity on a Risø airfoil that can stabilize the vortex generated in the cavity. 


\section{Penalized Vortex in Cell Method Implementation}

\subsection{Poisson Equation}

The first equation to be solved in the vortex in cell method is the Poisson equation. It is used to determine stream function from vorticity (6). The solver uses a compact fourth-order method using a 9-point stencil finite difference scheme. The derivation of this scheme is presented in detail in $[45,46]$. The idea lying behind this compact scheme is to utilize the fourth-order central scheme for the second derivative. The Poisson equation can be written as:

$$
\Delta \psi=\frac{\partial^{2}}{\partial x^{2}} \psi+\frac{\partial^{2}}{\partial y^{2}} \psi=\omega .
$$

The second derivative approximation for the $\mathrm{x}$ axis is represented as:

$$
\frac{\partial^{2}}{\partial x^{2}}=\left(h_{x}+\frac{h_{x}}{12} \delta_{x}\right)^{-1}+O\left(h^{4}\right)
$$

where $\delta$ is a central difference operator, $h$ represents the grid spacing and $x$ is the direction. The same formula applies to the $y$ axis. Rearranging the initial equation leads to a 9-point finite difference scheme.

$$
\begin{gathered}
\mathrm{a} \psi_{i j}+\mathrm{b}\left(\psi_{i+1, j}+\psi_{i-1, j}\right)+\mathrm{c}\left(\psi_{i, j+1}+\psi_{i, j-1}\right)+\mathrm{d}\left(\psi_{i+1, j+1}+\psi_{i-1, j-1}+\right. \\
\left.+\psi_{i+1, j-1}++\psi_{i-1, j+1}\right)=\frac{\Delta h_{x}^{2}}{2}\left(8 \omega_{i, j}+\omega_{i+1, j}+\omega_{i-1, j}+\omega_{i, j+1}+\omega_{i, j-1}\right),
\end{gathered}
$$

where the coefficients are: $\mathrm{a}=-10\left(1+\gamma^{2}\right), \mathrm{b}=5+\gamma^{2}, \mathrm{c}=\gamma^{2}-1, \mathrm{~d}=\left(1+\gamma^{2}\right) / 2$, $\gamma=h_{x} / h_{y}$.

\subsection{Velocity-Stream Function}

The next step that is necessary to be executed in the VIC method algorithm is determining the velocity from the stream function:

$$
u=\psi_{y}, \quad v=-\psi_{x}
$$

Similarly to the Poisson equation, the fourth-order compact discretization was utilizedin this case. To achieve higher-order, Hermite interpolation was used, in which the derivative was implicated in the form of a finite difference scheme. A noticeable feature of this scheme is the necessity of knowing the value at the boundary or using periodic boundary conditions. For the Neumann boundary condition, the scheme should be modified accordingly [47]. A more detailed description and derivations can be found in [48]. The problem for determining the velocity components $u$ and $v$ (12) requires solving a tridiagonal system of equations:

$$
\begin{array}{r}
4 \psi_{y,(i, j)}+\psi_{y,(i, j+1)}+\psi_{y(i, j-1)}=\frac{3}{h_{y}}\left(\psi_{i, j+1}-\psi_{i, j-1}\right), \\
4 \psi_{x,(i, j)}+\psi_{x,(i+1, j)}+\psi_{x(i-1, j)}=-\frac{3}{h_{x}}\left(\psi_{i+1, j}-\psi_{i-1, j}\right) .
\end{array}
$$

\subsection{Penalization}

Including the presence of solid irregular objects in a computational domain is usually troublesome. This algorithm is based on a very plain, yet tricky idea. We treat the entire numerical domain as a porous medium with zones of different properties. The fluid region has perfect permeability and the solid body is impermeable. The main advantage of this method is that the mesh does not have to fit the body boundary. Another advantage is that the no-slip boundary condition does not have to be defined and all calculations are carried out on regular grids without any need for modifying the system of equations.

This approach allows the fluid motion problem to be solved by solving the NavierStokes-Brinkman equation and, in fact, requires the addition of one more step to the entire algorithm. Therefore, we rely on a three-way split of the original equation: 
- Advection;

- Penalization;

- Diffusion.

The definition of the fluid and solid region is done by assigning a proper value of characteristic function $\chi$, which is equal to 1 in solids and 0 in fluids. Solid body porous flow properties are defined by a non-dimensional penalization parameter $\lambda=\mu \varphi H / \varrho k u_{\infty}$, where $k$ is the intrinsic permeability, $\mu$ the viscosity, $\varphi$ porosity, $H$ is body height, $\varrho$ density, and $\mathbf{u}_{\infty}$ is free-stream velocity.

The proper value of $\lambda$ coefficient is selected experimentally. It will be responsible for enforcing a no-slip boundary condition and the generation of vorticity on the wall. If it is chosen incorrectly, some residual slip velocity will exist. According to [49] $\lambda=10^{8}$ is a good choice that shows the best agreement in respect to reference solutions of flow past an impulsively started cylinder. To prevent a rapid change of the value of the characteristic function, a smooth transition function has been introduced. The length of the transition layer is defined as $\varepsilon=2 \sqrt{2} h$, Figure 1 . At the boundary of the solid wall, the value of the characteristic function $\chi$ is equal to 0.5 . The buffer layer function was inspired by [50] and is defined as:

$$
\chi=\left\{\begin{array}{cc}
0 & \text { for } x>\sqrt{2} h \\
\frac{1}{2}+\frac{1}{2} \cos \left(\pi\left(\frac{1}{2}+\frac{x}{2 \sqrt{2} h}\right)\right) & \text { for }-\sqrt{2} h<x \leq \sqrt{2} h \\
1 & \text { for } x<-\sqrt{2} h
\end{array}\right.
$$

where $x$ is the distance from the solid body wall. If the grid cell is inside the solid body, its distance from the wall is negative. This step requires the calculation of the distance from the grid node to the solid body surface. The presented solver allows the flow around an obstacle of any shape to be computed.
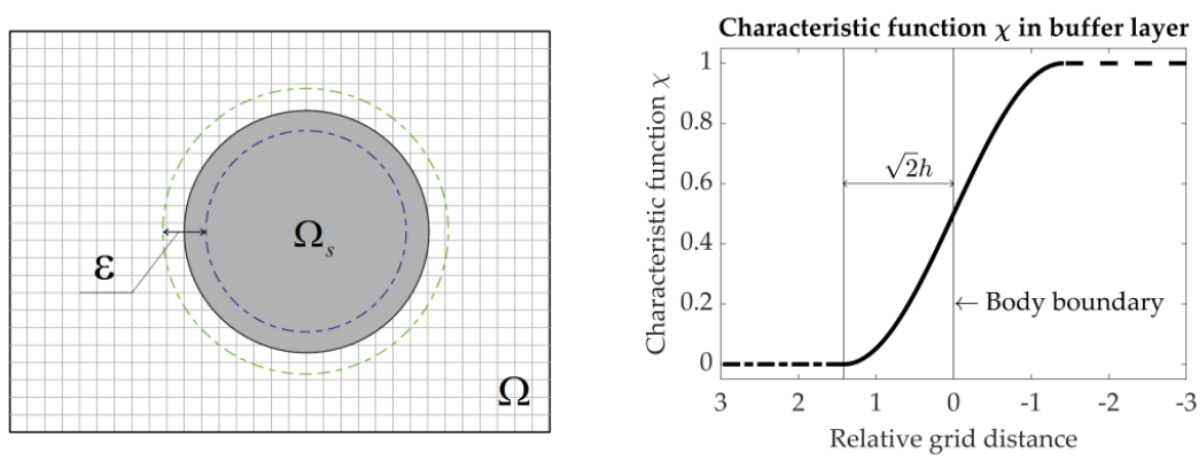

Figure 1. Definition of characteristic function $\chi$.

Preparation of the grid requires a solid body geometry prepared in CAD software and exported as a stereolithography file (STL), Figure 2a. This format represents the geometry as a surface shell consisting of unstructured triangles. Each triangle is described with 3 vertices and a normal vector pointing outward. The geometry is prepared as a 3 dimensional, symmetrically extruded prism. The total height of the prism should be bigger than at least 2 thicknesses of a transition layer $\varepsilon$. For the need of the algorithm, the $3 \mathrm{D}$ geometry file is used as an input because the volume of a body and triangular shell is required. 


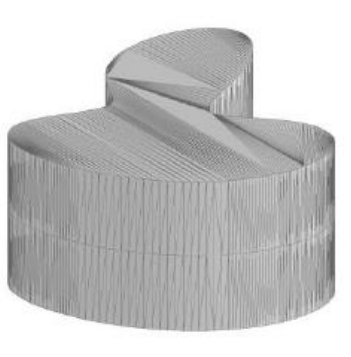

(a)

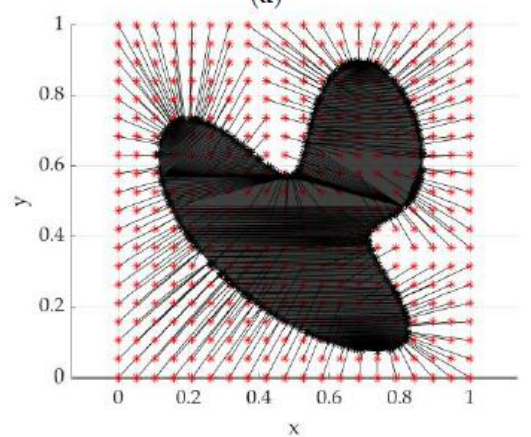

(c)

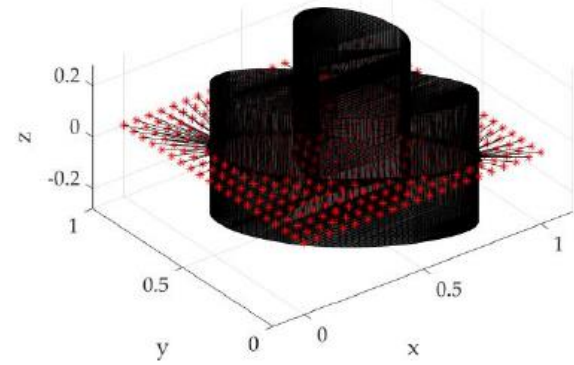

(b)

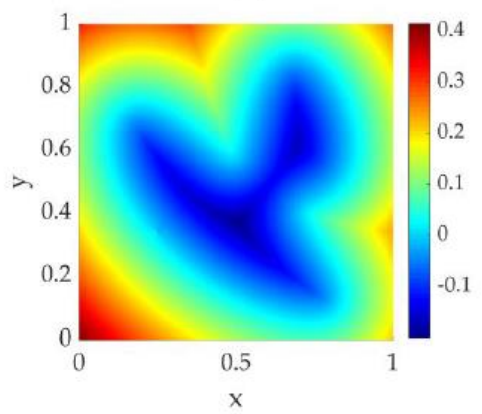

(d)

Figure 2. Algorithm for preparing input files for simulation: (a) Isometric view of exported STL geometry; (b) seeded grid points (red stars); (c) top view. For each point, the smallest distance is marked by a black line; (d) distance from the body. The negative distance can be observed by the points that are inside the volume of the body.

The algorithm is designed to find the distance between each grid point and the triangle of the surface shell. It is versatile and works regardless of the number of dimensions in which the calculations are carried out. For the simulation of two-dimensional flow, the points are distributed only on the plane that cut the body symmetrically, Figure $2 \mathrm{~b}$. If the point lays inside the volume of the body, the distance to triangular elements of the surface will be negative. If the distance is positive, then the point will belong to the fluid domain, Figure 2c,d. Matlab library that allows finding the closest distance of a point to a surface of a body represented as a set of triangles is point2trimesh [51]. Point2trimesh is based on the projection of a grid point on a triangle plane. After this projection, using barycentric coordinates, a 'point in triangle test' is done [52]. Each grid point is checked with every triangle in the geometry and the smallest distance is found. The algorithm for checking distance between a point $x$ and triangle created from vertices $\mathbf{A}, \mathbf{B}, \mathbf{C}$ can be presented in 8 steps as in Figure 3.

Preparing input files for the simulation is done only once and as a result, a set of files is generated. A separate file is generated for each subdomain, which is later used for parallel calculations. This approach is also well suited for three-dimensional calculations and the only change that has to be made is to seed points in space instead of distributing them on a plane. 


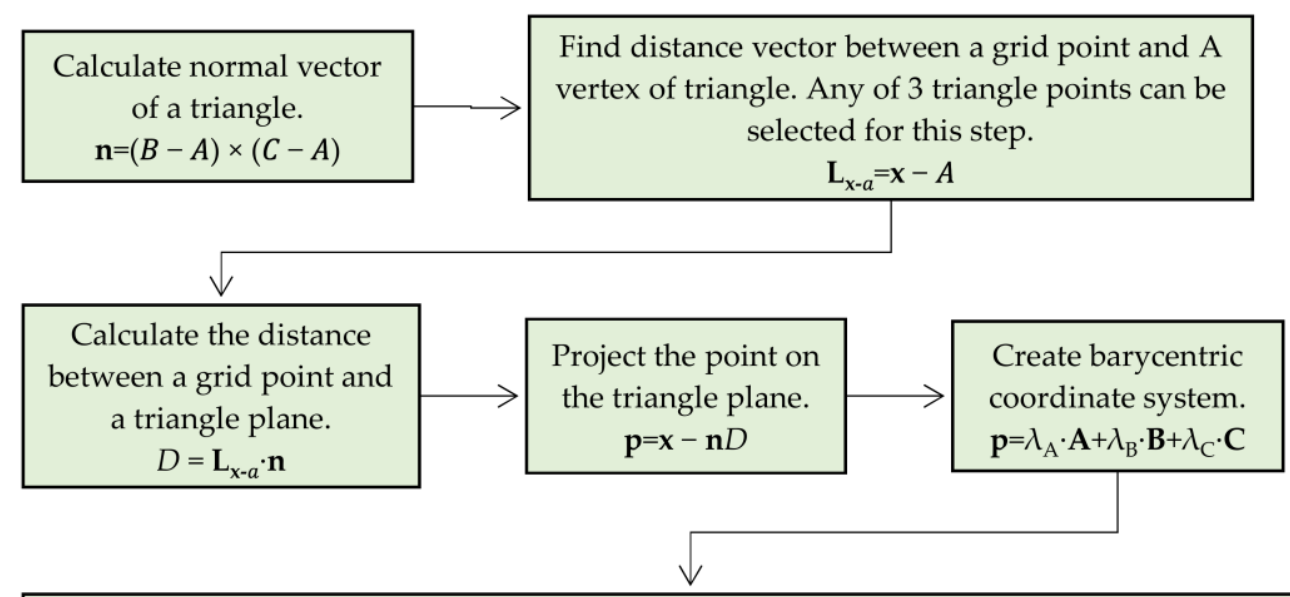

Check barycentric coordinate system coefficients. If all coefficients are bigger than 0 and smaller than 1 then a point is in a triangle. If one coefficient is equal to 0 and sum of the two others is equal to 1 then point lays on the edge. If one coefficient is equal to 1 and two remaining are equal to 0 then the point lays on the vertex. If any of the coefficients are bigger than 1 than point is not in triangle or lays on edge/vertex. If it is in triangle then $\mathrm{D}$ value from point no. 3 is the minimum distance.

Find smallest distance to all 3 edges and vertices of a triangle. Find minimum value obtained from point no. 6 and no. 7 .

Move to the next triangle and compare the distances against latter one. Check all triangles for a point and move to the next grid point.

Figure 3. Finding the distance of a point.

Once the characteristic function for the prepared geometry is obtained the penalization step can be done. It can be written that the continuous penalization of a velocity field is:

$$
\frac{\partial \mathbf{u}}{\partial t}=\chi \lambda \mathbf{u}
$$

In the following approach presented in [53], a first-order Euler scheme was used. The implicit formulation is unconditionally stable and the corrected velocity field is obtained with a simple expression:

$$
\mathbf{u}^{n+1}=\mathbf{u}^{n}-\left(\frac{\chi \lambda \Delta t \mathbf{u}^{n}}{1+\chi \lambda \Delta t}\right) .
$$

When the velocity correction term is known the change of vorticity field in time can be calculated:

$$
\frac{\partial \omega}{\partial t}=\nabla \times(\chi \lambda \mathbf{u})
$$

The correction of the vorticity is also done with a first-order approximation in respect to time. The curl operator was discretized with a fourth-order central scheme:

$$
\omega^{n+1}=\omega^{n}-\nabla \times\left(\frac{\chi \lambda \Delta t \mathbf{u}^{n}}{1+\chi \lambda \Delta t}\right) .
$$

\subsection{Advection and Remeshing}

The advection of vortex particles is solved using the fourth-order Runge-Kutta method. Each sub-step uses interpolated velocity values based on the 4 nearest nodes. The velocity field is the same for all sub-steps. Each particle starts from the node carrying its 
vorticity portion (vorticity value from Eulerian grid). After translation of the particles, the vorticity must be returned to the grid. Therefore, a remeshing procedure must be performed at each time step. The translated particle contributes its vorticity to neighboring nodes with the use of two one-dimensional kernels multiplied together. Therefore, vorticity at the node is the sum of all portions of the vorticity:

$$
\omega(x, y)=\sum_{p} \Gamma_{p} \varphi\left(\frac{x_{j}-x_{p}}{h}\right) \varphi\left(\frac{y_{j}-y_{p}}{h}\right) \frac{1}{h^{2}} .
$$

In Equation (12) $x_{p}, y_{p}$ denotes the particle Cartesian coordinate, $x_{j}, y_{j}$ grid coordinates and $\Gamma_{p}$ is particle circulation. Due to consistency preservation of the support (number of nodes to which the vorticity is redistributed) it has been decided to use the $Z_{2}$ cardinal spline interpolation kernel. In fact, it is the same as the popular $\mathrm{M}_{4}{ }^{\prime} \mathrm{B}$-spline kernel. For the interior of the domain, the following formula was used:

$$
Z_{2}= \begin{cases}1-\frac{5}{2} x^{2}+\frac{3}{2} x^{3} & \text { for } 0 \leq x \leq 1 \\ \frac{1}{2}(x-1)(x-2)^{2} & \text { for } 1<x \leq 2 \\ 0 & \text { for } x>2\end{cases}
$$

where $x$ denotes the normalized distance from the node. If a particle approach cell is adjacent to the boundary, special treatment is required to prevent redistributing the vorticity outside the domain.

Therefore, a one-sided scheme was derived in [54] to overcome this difficulty, see Figure 4. Particles located between a wall $(I=0)$ and the first unbounded row $(I=1)$ contributes their vorticity according to this formula:

$$
Z_{2}=\left\{\begin{array}{cc}
1-\frac{7}{6} x+\frac{1}{6} x^{2}+\frac{1}{3} x^{3} & \text { for } I=0 \\
\frac{4}{3}(x-1)+\frac{1}{3}(x-1)^{2}+\frac{2}{3}(x-1)^{3} & \text { for } I=1 \\
-\frac{1}{3}(x-2)+\frac{1}{6}(x-2)+\frac{1}{3}(x-2)^{3} & \text { for } I=2
\end{array}\right.
$$

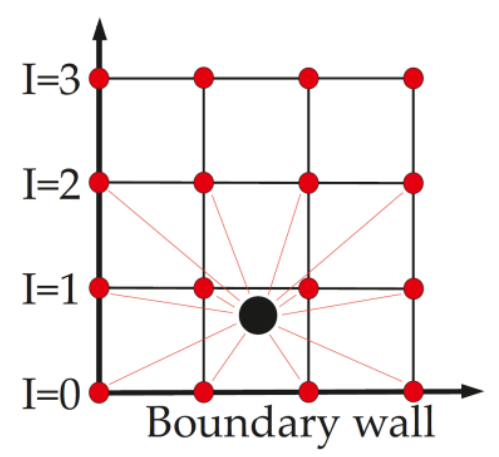

Figure 4. One side redistribution.

Redistribution in a tangential direction is the same as for the interior of the domain. As a result, for unbounded flow, we have a 16 point support and for the boundary a 12 point support. Improving the order of accuracy with more complex kernels [55] requires using at least a 6-point support stencil for each dimension. For the three-dimensional case, each particle must contribute its vorticity to 216 neighboring nodes, which is extremely computationally expensive. However, there are procedures [56], where remeshing is performed in only one-dimensional sub-steps, reducing the computational complexity to $O(6 d)$, where $d$ is dimensions number.

\subsection{Diffusion}

For the temporal discretization, the Crank-Nicholson explicit-implicit scheme was applied. It allows the second-order of discretization to be achieved over time. Substitution 
of the second derivative with the fourth-order central scheme for the second derivative $\delta_{x}^{2}\left(1+\frac{\delta_{x}^{2}}{12}\right)^{-1}$ and $\delta_{y}^{2}\left(1+\frac{\delta_{y}^{2}}{12}\right)^{-1}$ resulted in obtaining the fourth-order of accuracy in space. The result of this approach is a nine-point stencil system of linear equations both for LHS and RHS. The implementation of the factorization-based method [57] allowed the equation to be solved in two steps, with a 3-point stencil for the LHS and nine for the RHS. Each sub-step solves a diffusion equation in a separate direction. This method is unconditionally stable. The fourth-order of accuracy over time can be achieved through Richardson extrapolation by solving the diffusion equation three times, twice with $\Delta t / 2$ and once with $\Delta t$. A complete derivation and stability analysis of this implementation can be found in [58]. The initial Equation (20) and a ready-to-use two-step formulation (21) follows:

$$
\begin{gathered}
\frac{\omega^{n+1}-\omega^{n}}{\Delta t}=\frac{1}{2} v\left(\frac{\partial^{2}}{\partial x^{2}}\left(\omega^{n+1}+\omega^{n}\right)+\frac{\partial^{2}}{\partial y^{2}}\left(\omega^{n+1}+\omega^{n}\right)\right) \\
\mathrm{a} \omega_{i, j}^{*}+\mathrm{b}\left(\omega_{i+1, j}^{*}+\omega_{i-1, j}^{*}\right)=\mathrm{c} \omega_{i, j}^{n}+\mathrm{d}\left(\omega_{i+1, j}^{n}+\omega_{i-1, j}^{n}+\omega_{i, j+1}^{n}+\omega_{i, j-1}^{n}\right)+ \\
+\mathrm{e}\left(\omega_{i+1, j+1}^{n}+\omega_{i+1, j-1}^{n}+\omega_{i-1, j+1}^{n}+\omega_{i-1, j-1}^{n}\right), \\
\mathrm{a} \omega_{i, j}^{n+1}+\mathrm{b}\left(\omega_{i, j+1}^{n+1}+\omega_{i, j-1}^{n+1}\right)=\omega_{i, j}^{*}
\end{gathered}
$$

where $\mathrm{a}=2 r+\frac{5}{6}, \mathrm{~b}=-r+\frac{1}{12}, \mathrm{c}=\left(-2 r+\frac{5}{6}\right)^{2}, \mathrm{~d}=\left(-2 r+\frac{5}{6}\right)\left(r+\frac{1}{12}\right), \mathrm{e}=\left(r+\frac{1}{12}\right)^{2}$, $r=\frac{v \Delta \mathrm{t}}{2 h^{2}}, \omega_{i, j}^{*}$-the result of the first sub-step.

\subsection{Force Evaluation}

For a detached bluff body flow, this formula can be used as long as the vorticity and its normal derivatives are accurately captured at the boundary. An evaluation of the acting forces on a body is critical when dealing with aerodynamics. Doing it quickly and accurately is often a challenge when neither pressure field nor normal vectors to the wall are known. Since this algorithm relies on the penalization method and the mesh is not adjusted to fit the body, an alternative approach must be chosen. The lift and drag force can be evaluated by the measurement of the change of momentum in the computational domain. Following [41,59], we integrated the penalized velocity over the body surface obtaining the change of momentum. The change of momentum in time gives the acting force. It can be written that the change of momentum is equal to:

$$
\Delta M=\int_{\text {Body }}\left(\mathbf{u}-\mathbf{u}_{\text {Body }}\right) d \mathbf{x}
$$

In each iteration, some part of the fluid enters the solid body resulting in a velocity in the solid. The penalization step forces the removal of velocity $\mathbf{u}$ from the solid domain so that it becomes $\mathbf{u}_{\text {Body }}$ (velocity inside the body, $\mathbf{u}_{\text {Body }} \approx 0$ ). The difference between those two values is the amount of penalization that was performed on the body. The formula to express the acting force is then:

$$
\boldsymbol{F}=\frac{d}{d t} \int_{\text {Body }} \mathbf{u}-\mathbf{u}_{\text {Body }} d \mathbf{x}=\frac{\Delta M}{\Delta t} .
$$

In practice, the entire computational domain can be integrated because fluid and solid regions are distinguished by their characteristic function $\chi$. The ready-to-use formula is:

$$
\boldsymbol{F}=-\int_{\Omega} \frac{\lambda \chi \mathbf{u}^{n}}{1+\lambda \chi \Delta t} d \mathbf{x}
$$


The lift and drag force coefficients were calculated with respect to their characteristic dimension:

$$
\begin{aligned}
& C_{D}=\frac{F_{x}}{p_{d} A}=\frac{F_{x}}{0.5 \rho u_{\infty}{ }^{2} d L}, \\
& C_{L}=\frac{F_{y}}{p_{d} A}=\frac{F_{y}}{0.5 \rho u_{\infty}{ }^{2} d L},
\end{aligned}
$$

where $p_{d}$ is dynamic pressure and $A$ is the reference area (characteristic dimension $d$ times reference length $L$ ). An alternative solution proposed by [60] or by [49] can be adopted.

\subsection{Boundary Conditions}

When dealing with unbounded flows such as flow past an airfoil or an impulsively started cylinder, it is necessary to prepare an adequate composition of the domain, assuring that the far-field condition is fulfilled. This results in the expansion of the computational domain size and the increase in the number of grid points that need to be calculated. While utilizing the vorticity-stream function formulation (Poisson equation), when the Neumann boundary condition is used, the velocity outflow angle is usually perpendicular to the outlet. For the inlet, outlet, top, and bottom walls, a Dirichlet boundary condition is applied. In solving the Poisson equation, the following functions were used: $\psi_{\text {Inlet }}=y$, $\psi_{\text {Outlet }}=y$, $\psi_{\text {Top }}=1, \psi_{\text {Bottom }}=0$.

A solid body is required to be placed at such a distance from the boundary that it does not interact with it. If the calculations are carried out on a uniformly spaced grid without adaptive mesh refinement [37] it is difficult to maintain body resolution and the proper grid points per boundary layer thickness [61], while keeping the problem size and calculation time within a reasonable range.

A remedy to this problem might be adding a correction term to the value of the Poisson boundary condition. Based on the vorticity distribution in the domain, the presence of a solid body can be included. This is because the body is the only source of vorticity and its concentration is the greatest in its vicinity. The correction term for the boundary condition of a domain can be approximated with the use of the Green function. For the 2D case, the expression is:

$$
\psi_{b}\left(x_{b}, y_{b}\right)=-\frac{1}{2 \pi} \sum_{p}^{N} \Gamma_{p} \ln \left(\sqrt{\left(x_{p}-x_{b}\right)^{2}+\left(y_{p}-y_{b}\right)^{2}}\right),
$$

where $\Gamma_{p}$-particle circulation, $x_{p}, y_{p}$-particle position, $x_{b}, y_{b}$-boundary point position, and $N$-particle count.

Since the complexity of this step is $O\left(N N_{b}\right)$, for a larger number of particles, fast multipole methods should be applied, thereby reducing the cost to $O(N \log N)$ (for calculating the stream function for the entire domain) [62]. However, implementing this method in parallel computation might be difficult. Alternatively, from the observation that the stream function on boundaries is smooth and does not change rapidly, interpolation could be applied to reduce the amount of calculation required. Using cubic spline interpolation, which was presented in the papers of Lee $[63,64]$. This reduces the number of operations to $O\left(M_{b} N / P\right)$, where $M_{b}$ is the number of interpolation nodes on the boundary, $P$ is the number of processors used for the calculation if parallel computation is employed.

Additionally, since the values of the stream function do not change a lot with each iteration and if even more computational time reduction is needed, updating the stream function boundary values could be performed every few iterations. The justification for such a solution is that due to the splitting algorithm temporal accuracy is $O(\Delta t)$, and this forces the use of a very small step time size in order to maintain the proper level of overall accuracy. Thus, the change of velocity and vorticity field is also very small. The update boundary refresh rate would only depend on the demanded level of accuracy and should be selected empirically. 
Another advantage of using the corrected Dirichlet boundary condition is that the tangent velocity component will occur according to the local vorticity field distribution. This is highly desirable when we want to include the influence of an obstacle near the inlet, or, slightly deflect streamlines at the outlet (due to the presence of an airfoil at a certain angle of attack). On the other hand, a large vorticity flux at the outlet might lead to distortion of the velocity field and if some vortices of high strength leave the domain they will have a great impact on the stream function value at the boundary. As a result, a local, unnatural increase of velocity might occur. Therefore, the corrected Dirichlet boundary for the outlet should only be used if the vorticity at the domain outlet is properly diffused, or, some sort of outlet vorticity absorption filters are utilized.

\subsection{Solving Systems of Linear Equations}

The entire code was written in the $C$ language. To solve systems of linear equations (Poisson equation, velocity-stream function, and diffusion equation) with the use of MPI on multiple processors, the hypre library was employed. The library uses common preconditioning iterative methods to solve problems. This includes preconditioning methods for non-symmetrical systems such as GMRES and methods for symmetric matrices such as the conjugate gradient method. The hypre library uses $O(N)$ computations to solve a linear system with $\mathrm{N}$ variables and has been successfully tested on massively parallel machines with over 100,000 cores, with the largest problem of 12 billion unknowns [44].

All calculations are carried out using the interface for the structural mesh in which the spacing between the adjacent nodes of the computational mesh is the same. However, the hypre library allows efficient calculations for semi-structured and unstructured meshes to be performed. The semi-structural mesh means that certain areas of the computational mesh are not fully structural. They are characterized, for example, by a set of structural mesh blocks of different sizes glued together. Such an interface can be effectively combined with adaptive mesh refinement (AMR).

The hypre library was chosen for solving systems of linear equations mainly due to its intuitiveness, extensive documentation, as well as constant updates, and the introduction of new functions by the authors. It is also worth mentioning that the scalability and speed of calculations speak in their favor and are proven in numerous publications $[44,65]$. It was proven to maintain scalability up to 100,000 cores and the complexity of multigrid solvers is of the order $O(N)$. Scalability is understood as the ability to expand the computational domain by refining the mesh, adding new processes with no or a negligible drop in performance. In other words, as the number of processes with the same local problem size increases, (e.g., 1 process with an $8 \times 8$ grid and 4 processes with an $8 \times 8$ grid) the computation time remains the same. Good scalability can be understood as the efficiency of communication between processes. The less information between processes is exchanged and it is done more efficiently, the better the scalability will be. The solvers used in this implementation have been put in Table 1 . All systems of equations are in the form $\mathbf{A x}=\mathbf{b}$.

Table 1. Linear system of equations-solver selection.

\begin{tabular}{ccccc}
\hline Equation & $\begin{array}{c}\text { A Matrix } \\
\text { Diagonals }\end{array}$ & Type of Method & Solver & Preconditioner \\
\hline Poisson eq. & 9 & iterative & GMRES & PFMG V(1,1) \\
Velocity-stream func. & $2 \times 3$ & direct & CycRed & - \\
Diffusion eq. & $2 \times 3$ & direct & CycRed & - \\
\hline
\end{tabular}

The best solver selection was based on test runs with different setups. For solving the Poisson equation, the fastest solver was GMRES, with a parallel semicoarsening multigrid (PFMG). A default $\mathrm{V}$ cycle with 1 relaxation and 1 smoothing sweep was used. No significant difference was observed when the number of relaxation and sweeping cycles was changed. A default weighted Jacobi smoother was used. Using a red/black GaussSeidel smoother led to no improvement in performance. An acceptable performance was 
also obtained with the use of a PFMG and SMG solver with no preconditioning. Regardless of preconditioning, the conjugated gradient-based solvers solution times were almost twice the time of the GMRES solver.

For the velocity-stream function and the diffusion equation, a direct solver based on cyclic reduction was chosen. This is the only solver that allows 1D problems embedded in a D-dimensional grid to be solved. For the tridiagonal system of linear equations, the time needed for solving the problem was reduced approximately by 10 times in comparison to the iterative method.

\section{Test Problem-Impulsively Started Cylinder}

Accuracy tests of pure high-order vortex in cell method were already performed in $[39,40]$. In those papers, Green-Taylor vortex and flow in the lid-driven cavity were investigated. These tests showed impressive agreement with reference results. To evaluate the accuracy of the penalization method, a classical benchmark case of a flow past an impulsively started cylinder was chosen. This is one of the most popular tests for an unsteady simulation for low and moderate Reynolds numbers. The tests were performed for three Reynolds numbers: 550, 3000, and 9500. The selection of numbers was based on the wide accessibility to results from other researchers. Through the simulation drag coefficient change in time was measured. It was calculated with Equations (24) and (25). Simulations have been performed in a rectangular box with dimensions of $L_{x} \times L_{y}=1 \times 0.5$ and a cylinder diameter of $D=0.1$. Grid resolution varied with the Reynolds number. The cylinder is centered at $(x, y)=(0.4,0.25)$. A grid convergence study showed that it is required to maintain approximately 10 cells in the boundary layer. The boundary layer thickness was estimated with the formula $\delta \approx D / \sqrt{R e}$. A similar number of cells in the boundary layer needed for convergence can also be observed in [49]. This led to the problem size of the dimensions $2400 \times 1200,4800 \times 2400,9600 \times 4800$, respectively, and $h$ equal to $1 / 2400,1 / 4800$, and $1 / 9600$. This translates to 240,480 , and 960 cells across the cylinder $(D / h)$. The grid size details for the converged solutions are presented in Table 2.

Table 2. Grid sizing details.

\begin{tabular}{cccc}
\hline $\operatorname{Re}$ & $\delta$ & Grid Size $\boldsymbol{h}$ & Cells per Boundary Layer \\
\hline 550 & $\sim 0.040$ & $\sim 0.0040$ & 10.2 \\
3000 & $\sim 0.002$ & $\sim 0.0002$ & 8.8 \\
9500 & $\sim 0.001$ & $\sim 0.0001$ & 9.9 \\
\hline
\end{tabular}

To reduce computational costs for the workstation, the domain was divided into $N_{x} \times N_{y}=24 \times 12$ subdomains. Running more processes than the physical availability of processor core count significantly increases the efficiency of the hypre library (solving systems of linear equations). For the Poisson equation, the tolerance criterion was set to $10^{-8}$, which led to approximately 3 iterations to solve this equation with the GMRES solver. The timestep for all cases was related with the grid size by the formula $\Delta t=0.5 \mathrm{~h} / u_{\infty}$, $\mathbf{u}_{\infty}=\left(u_{\infty}, v_{\infty}\right)=(1,0)$. This results in the maximum value of the CFL number being slightly below unity for the entire simulation time. The Reynolds number was modified with the value of the viscosity. The obtained results were compared against $[61,66]$. Apart from the drag coefficient evolution in time, streamlines and vorticity distribution were compared against $[64,67]$.

The results of the computation show good agreement with the reference data, Figure 5a-c. Time was presented as a nondimensional number with the formula $\tau=t \cdot u_{\infty} / D$. The penalized vortex method data correspond to the results of other authors even though a moderate value of grid and time step were chosen. 


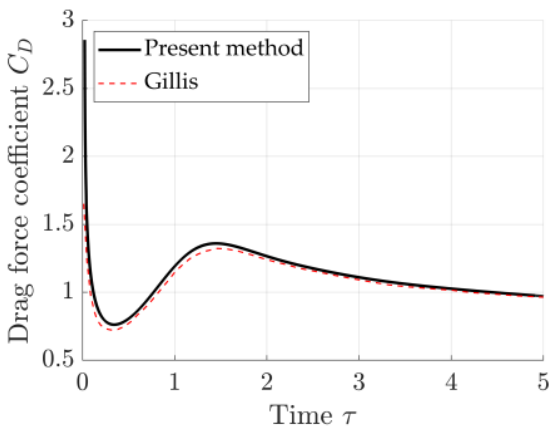

(a)

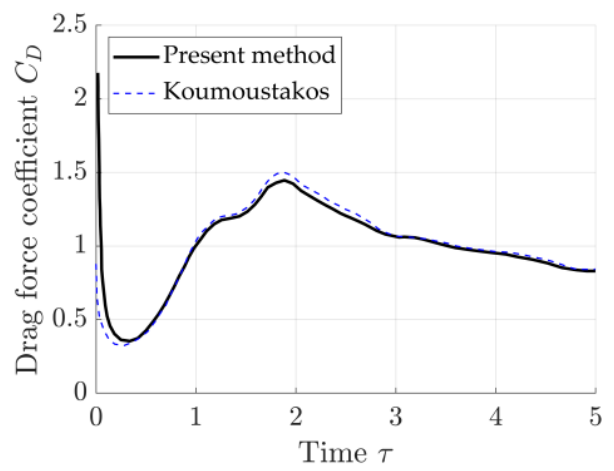

(b)

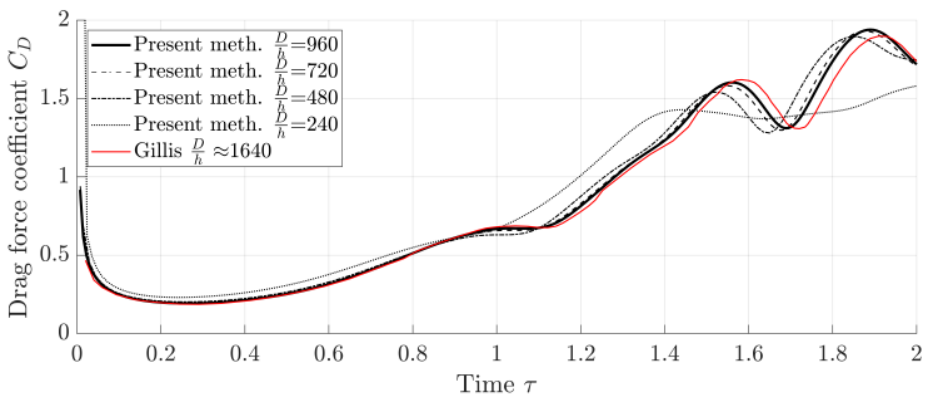

(c)

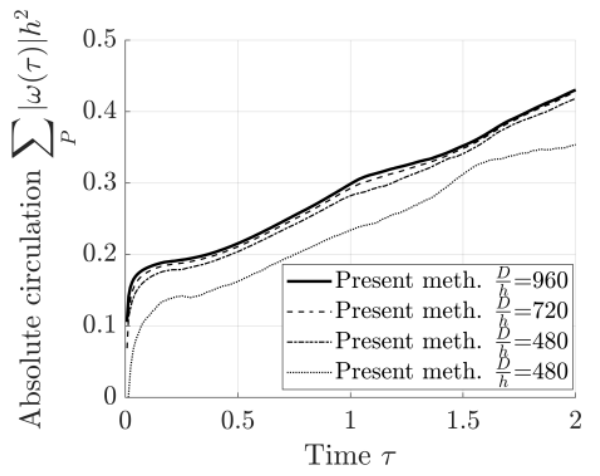

(d)

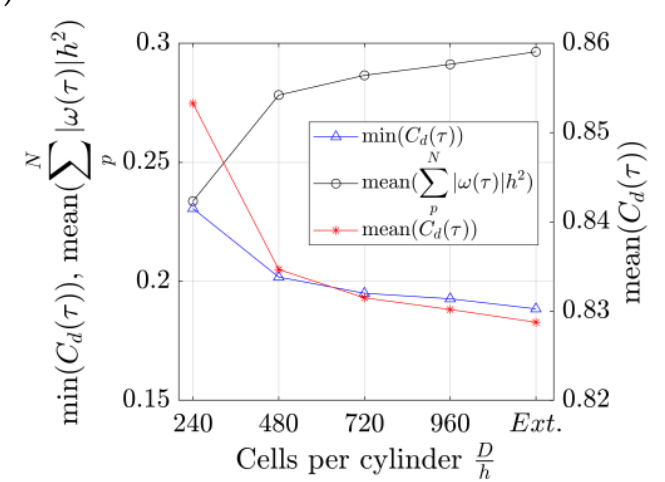

(e)

Figure 5. Drag coefficient change in time for: (a) $R e=550$ [61]; (b) $R e=3000$ [66]; (c) $R e=9500$ [61]; (d) absolute circulation convergence; (e) statistical functions convergence.

The main concern regarding lack of accuracy in time seems to be irrelevant. Even though accuracy is reduced due to splitting and implicit Euler penalization, the shape of the drag coefficient curve does not seem to be shifted in time and no lagging was observed. The values were also predicted precisely. For the case with the highest Reynolds number, $R e=9500$, nearly no dissimilarities can be noticed. Results were in agreement both with the values and time.

For this Reynolds number, a grid independence test was done. Four simulations were done to determine how the solution was changing when grid resolution was increased. The simulation started with a coarse grid with 240 cells along the cylinder diameter, Figure 5c. The coarse grid function does not fit the reference data. Refining the mesh by a factor of 2 $(r=2, \mathrm{D} / \mathrm{h}=480)$ allowed the solution to be improved. Running simulation with the fine grid $(D / h=960)$ allowed acceptable independence of the grid resolution to be achieved. There is nearly no change in respect to the results from the intermediate grid $(D / h=720)$.

Besides the drag coefficient, the absolute global value of circulation transported by vortex particles was measured, Figure $5 \mathrm{~d}$. As time increases, the global absolute amount of vorticity grows. The grid independence test shows that the solution is converging when the grid is refined. To measure the rate of convergence some statistical functions $f$ are 
needed. The minimum and mean values of drag and the mean value of absolute particles' vorticity were taken for each grid resolution. The collected data and extrapolated values of functions (marked as Ext.) can be seen in Figure 5e.

The value obtained from the Richardson extrapolation is close to one, where the solution is fully independent of the grid resolution and timestep size $\left(f_{h=0}\right)$. In order to calculate this value, an observed order of convergence $p$ is needed. Three solutions $f_{\text {coarse, }}$, $f_{\text {medium }}$ and $f_{\text {fine }}$ are necessary. The grid in each case is refined by a factor of $r$. The order can be calculated with the equation:

$$
p=\frac{\ln \left(\frac{f_{\text {coarse }}-f_{\text {medium }}}{f_{\text {medium }}-f_{\text {fine }}}\right)}{\ln (r)} .
$$

The Calculated order is then used for the Richarson extrapolation:

$$
f_{h=0}=f_{\text {fine }}-\frac{f_{\text {fine }}-f_{\text {medium }}}{\mathrm{r}^{p}-1} .
$$

Apart from the close to an exact value, a good way to measure how well the grid is resolved, a recommended practice is to calculate the grid convergence index (GCI). This is a percentage measurement of convergence based on safety factor $\left(F_{S}=1.25\right)$, the relative difference of two solutions $\varepsilon$, refinement and the order of convergence:

$$
G C I_{\text {fine }}=\mathrm{F}_{\mathrm{s}} \frac{|\varepsilon|}{\mathrm{r}^{p}-1}=\mathrm{F}_{\mathrm{s}} \frac{\left|\frac{f_{\text {fine }}-f_{\text {medium }}}{f_{\text {fine }}}\right|}{\mathrm{r}^{p}-1}
$$

When this index is calculated, the refinement level needed to achieve the desired level of grid convergence index $\left(\mathrm{GCI}^{*}\right)$ can be evaluated:

$$
r^{*}=\left(\frac{G C I_{\text {fine }}}{G C I^{*}}\right)^{\frac{1}{p}}
$$

In Table 3 the values obtained from the analysis are presented. One can see good convergence properties. The values of functions for the fine grid are close to the one obtained from Richardson extrapolation. The GCI for fine mesh is at an acceptable level. If the grid were to be further refined, the maximum change in the results should be expected

\begin{tabular}{|c|c|c|c|c|c|c|c|c|}
\hline Function $f$ & p & $f_{\text {fine }}$ & $f_{h=0}$ & $G C I_{\text {fine }}$ & GCI $I_{\text {coarse }}$ & $\frac{\text { GCIcoarse }}{r^{p} G \text { CIfine }}$ & $G C I^{*}$ & $r^{*}$ \\
\hline $\min \left(C_{D}(\tau)\right)$ & 1.66 & 0.193 & 0.189 & $2.7 \%$ & $8.3 \%$ & 0.96 & $0.5 \%$ & 2.79 \\
\hline $\begin{array}{c}\operatorname{mean}\left(C_{D}(\tau)\right) \\
\text { Mean }\end{array}$ & 2.05 & 0.830 & 0.829 & $0.2 \%$ & $0.9 \%$ & 1 & $0.2 \%$ & 1.04 \\
\hline$\left(\sum_{p}^{N}|\omega| h^{2}\right)$ & 1.79 & 0.291 & 0.297 & $2.3 \%$ & $8.1 \%$ & 1.05 & $0.5 \%$ & 2.33 \\
\hline \multicolumn{9}{|c|}{ Wpisz tutaj równanie. } \\
\hline
\end{tabular}
for peak values. In addition, the total absolute vorticity is still susceptible to change of the grid resolution.

Table 3. Grid convergence analysis.

To state if the solution is prone to converge asymptotically, the expression $\frac{G C \text { Icoarse }}{r^{p} \text { GCIfine }}$ should be around 1. From the results, it can be seen that this criterion is fulfilled. Obtaining GCI equal to $0.5 \%$ requires a refinement of the mesh by a factor of 3 .

The next step in the validation of the penalized vortex method implementation is the comparison of the streamlines and flow patterns that are developed in the test case of an impulsively started cylinder, Figure 6. Based on the experimental visualization results of Bouard and Coutanceau, the transient behavior of fluid can be examined. Depending on the fluid type (oil or water) the authors used two types of solid tracers, reflective 
(magnesium) and diffusive (Rilsan/polyamide microparticles). Their density is close to the fluid medium and thus, a homogenous mixture can be created. Once the carriage with a cylinder and camera mounted on it started to move, with the camera aperture being opened while moving, particles suspended in the fluid form streamlines-which can be seen in the photographs.

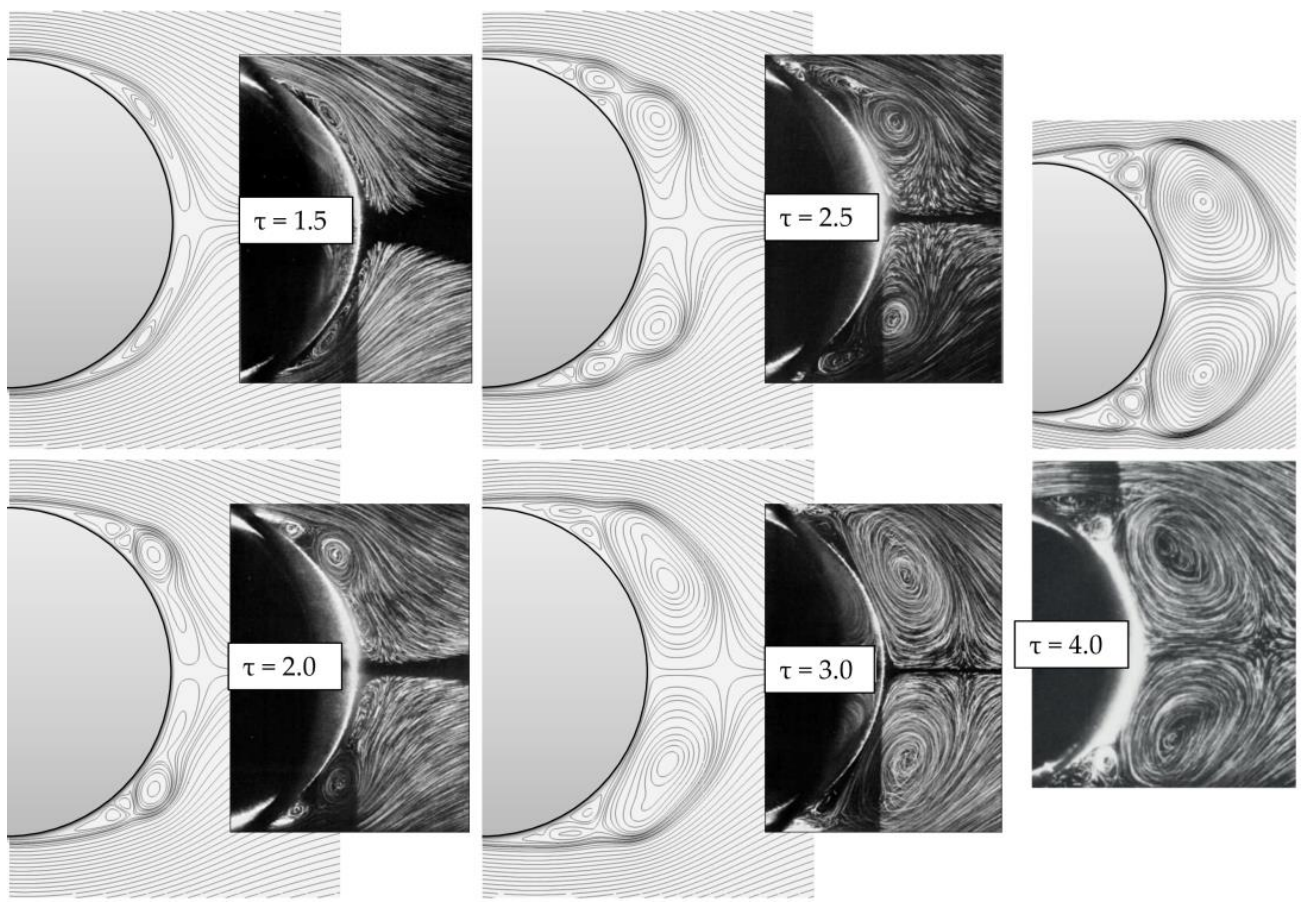

Figure 6. Streamline obtained from experiment [67] and numerical simulation for $R e=9500$.

The first sequence at $\tau=1.5$ shows a small recirculating wake that fits closely to the cylinder. Soon it evolves and grows. At $\tau=2$ nucleation of a smaller vortex core can be noticed. At $\tau=2.5$ the main vortex is separated into two parts. Subsequently, the main vortex grows even larger and space occupied by smaller eddies is absorbed by the main vertical structure. The main wake at $\tau=4$ grows to its nominal dimension and after that, it gets separated from the cylinder. Two smaller structures can still be observed.

The numerical results are in excellent agreement with the visualization. The flow regime was captured accurately, with scale and fine details preserved. For other Reynolds numbers, the visualizations were also consistent.

For such a high Reynolds number, the flow is unstable and the shedding generates complex vortex pairings. The flow regime at the Reynolds number equal to $R e=9500$ is dominated by convection, therefore the unsteady vorticity field will consist of fine-scale eddies and it should change dynamically with time. Figure 7 presents a comparison of the vorticity fields with the results from [64]. The left side with a grey background refers to the present results, the white background is a reference solution. Lee et al. also utilized the vortex penalized method, so no significant difference can be observed. The contours correspond exactly to the time frame, meaning that this implementation is accurate enough to solve more challenging cases. 


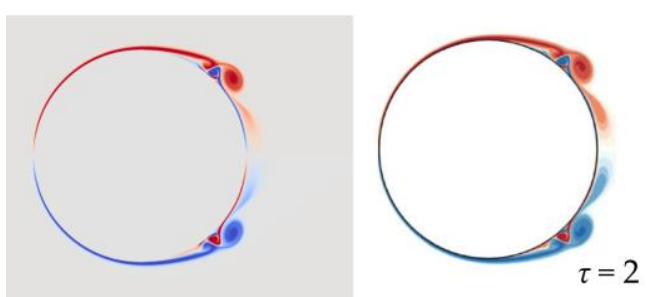

(a)

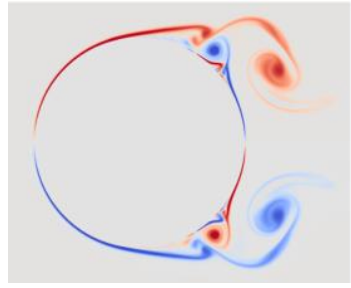

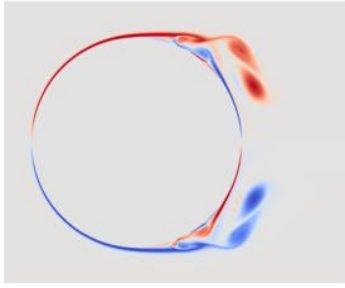

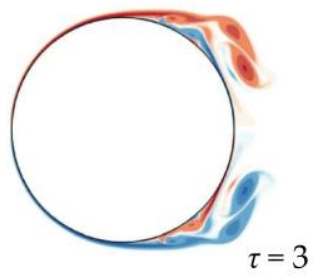

(b)

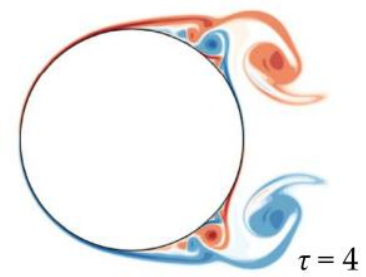

(c)

Figure 7. Vorticity evolution for impulsively started cylinder at $\operatorname{Re}=9500$ : (a) $\tau=2$; (b) $\tau=3$; (c) $\tau=4$.

\section{Numerical Simulation}

Flow past an airfoil with a cavity on its upper case is a very interesting case from the point of fluid mechanics. For calculation 3, geometries were tested for various angles of attack. Each geometry base on a Risø B1-18 airfoil and optimized cavities was reproduced from papers [28,29]. The shape of the geometry is presented in Figure 8. Looking from the top of this figure, the first one, a smooth profile, without any cavity, is described as a "Base" airfoil. The second one, with a sharp triangular cavity, is marked as "Cavity A". The airfoil with a big round cavity is named "Cavity B". The computation was done for the Reynolds number (based on the airfoil chord) equal to $R e=2 \times 10^{4}$. The tested angles of attack were 3,6 , and 9 degrees.

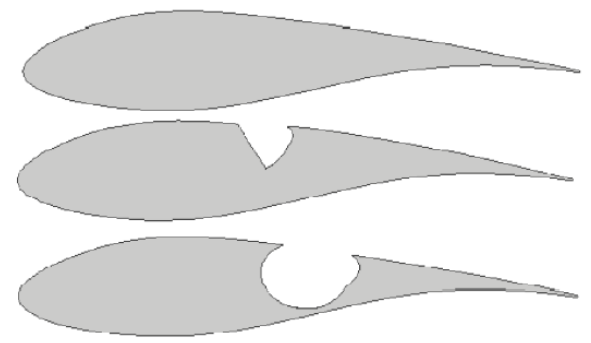

Figure 8. Geometries prepared for numerical simulation, from the top: standard Risø airfoil, airfoil with cavity A, airfoil with cavity B.

The following approach was based on the boundary layer cell count from Section 4, the grid size was equal to $14,400 \times 7700$ for all cases. The domain is a rectangle with dimensions $L_{x} \times L_{y}=1 \times 0.5$ and with the chord equal to $c=0.14$. This translates to a boundary layer cell count equal to 21 cells. The resolution was increased due to the need for an accurate representation of fine airfoil details such as a trailing edge or sharp edges of the cavity. The timestep size used was equal to $\Delta t=0.00002 \mathrm{~s}$. The domain composition is presented in Figure 9a. Here, an exemplary image of the vorticity distribution of cavity A is shown. Figure $9 \mathrm{~b}$ shows the resolution of the grid with a sharp edge of a cavity. 


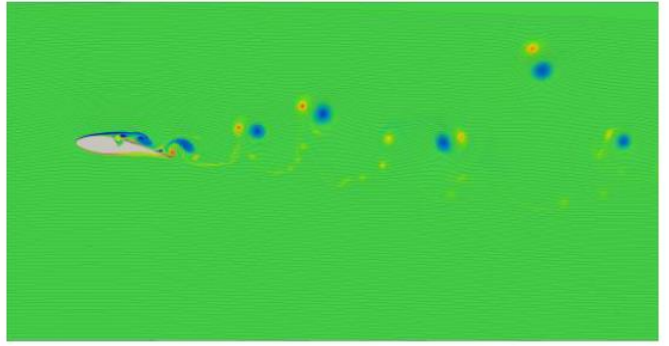

(a)

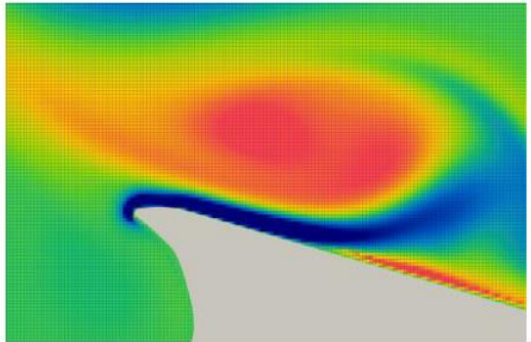

(b)

Figure 9. Numerical simulation: (a) Domain composition, (b) details of the mesh.

To minimize calculation time the domain was divided into $N_{x} \times N_{y}=48 \times 24$ subdomains. All cases were computed with the use of a cluster located at the Wroclaw Centre for Networking and Supercomputing.

To be able to evaluate the performance of the airfoil modification lift and drag coefficients were computed during the simulation. For the evaluation, an average value from a developed simulation was taken. The flow was treated as developed when the vorticity started exiting the computational domain i.e., $\tau=10$. In Table 4 the results from the calculation were presented. Due to its low performance, the case with cavity B was only calculated for the angle of attack $\alpha=6^{\circ}$.

Table 4. Comparison of lift and drag coefficients for different cases.

\begin{tabular}{ccccccccccccc}
\hline Case & $\alpha$ & $C_{L}$ & $C_{D}$ & $C_{L} / C_{D}$ & $\alpha$ & $C_{L}$ & $C_{D}$ & $C_{L} / C_{D}$ & $\alpha$ & $C_{L}$ & $C_{D}$ & $C_{L} / C_{D}$ \\
\hline Base & 3 & 0.64 & 0.85 & 7.52 & 6 & 0.88 & 0.105 & 8.35 & 9 & 1.17 & 0.148 & 7.87 \\
Cav.A & 3 & 0.66 & 0.093 & 7.09 & 6 & 0.95 & 0.116 & 8.20 & 9 & 1.27 & 0.152 & 8.37 \\
Cav.B & 3 & - & - & - & 6 & 0.89 & 0.139 & 6.40 & 9 & - & - & - \\
\hline
\end{tabular}

In all analyzed cases cavity $\mathrm{A}$ and cavity B on the upper surface of an airfoil lead to an increase of lift coefficient. The presence of a cavity deteriorates the $C_{L} / C_{D}$ ratio for $\alpha=3^{\circ}$ and $\alpha=6^{\circ}$. For $\alpha=9^{\circ}$ cavity A outperforms the smooth profile $-C_{L} / C_{D}$ ratio is higher for the airfoil with cavity A than for the standard Risø airfoil. For the base geometry, a noticeable increase in lift coefficient was observed. The drag coefficient is higher for the profile with a cavity in respect to the smooth profile in all cases and it increases with the increase of the angle of attack. For cavity A and $\alpha=9^{\circ}$, the drag coefficient increases to a value of $C_{D}=0.152$. This means that the $C_{L} / C_{D}$ ratio was increased by $6.4 \%$. Additionally, it should be noticed that the optimal angle of attack moved toward higher values.

Flows at such high Reynolds numbers start to fluctuate and the parameters that characterize airfoil performance change dramatically in time. Figure $10 \mathrm{a}, \mathrm{b}$ shows the $C_{L}$ and $C_{D}$ comparison between the smooth, base profile and one with the A-type cavity. The red line represents the smooth profile and the blue line is the cavity. The bold line shows the filtered signal based on local regression using weighted linear least squares and a 2nd-degree polynomial model.

The signal waveform is mainly represented by low-frequency oscillations. The signal spectral analysis showed that no dominant frequency was present. Based on the standard deviation value for developed flow, it can be said that both lift and drag coefficients tended to oscillate more for the case with the cavity. The calculated standard deviations are 0.149 and 0.172 for base and cavity, respectively. The course of filtered function for the lift coefficient shows that the airfoil with a cavity is superior in this field. 


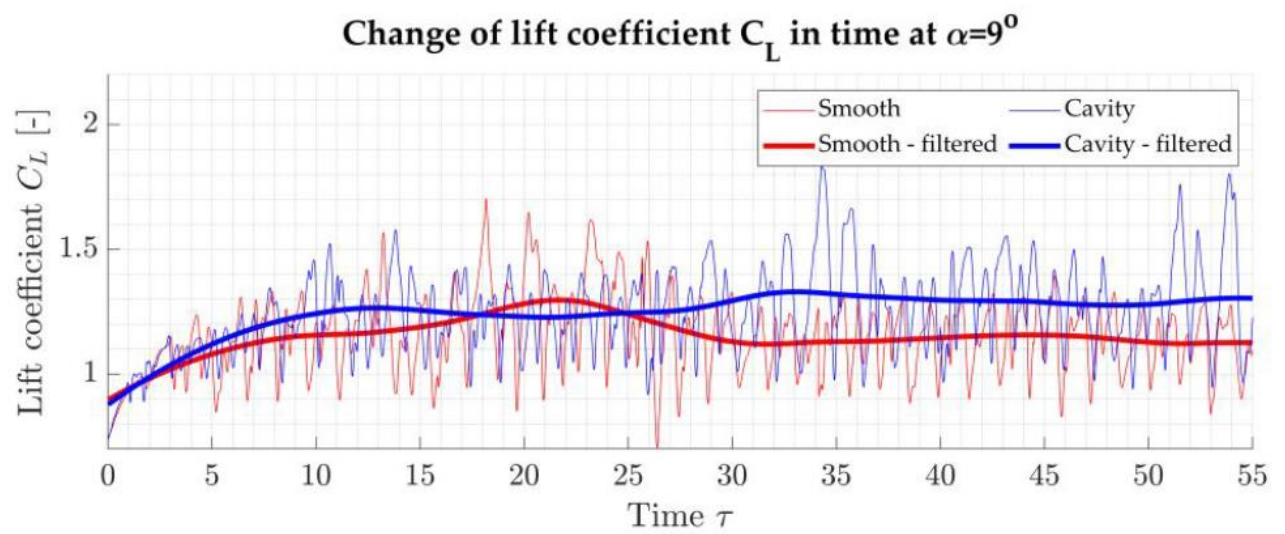

(a)

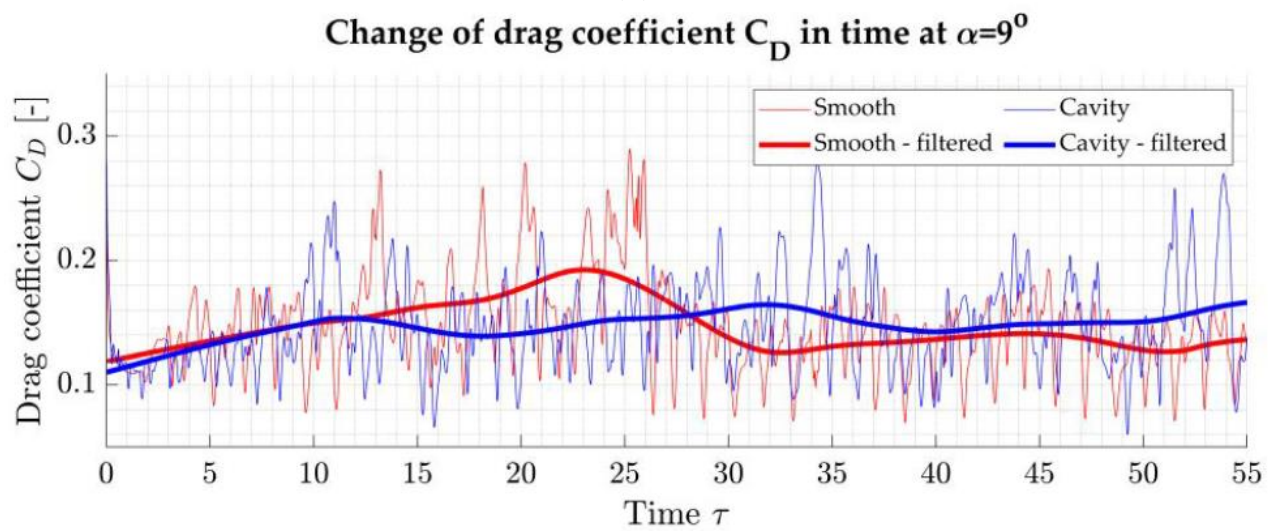

(b)

Figure 10. Lift and drag coefficient change in time for Risø airfoil with Cavity A at $\alpha=9^{\circ}$ : (a) lift coefficient; (b) drag coefficient.

Apart from the comparison of the $C_{L}$ and $C_{D}$ waveforms, a lot of information about the flowfield can be obtained during post-processing. Table 5 presents results from measurements of the recirculation zone and cavity zone. Here, the size and total vorticity from all cells were presented. The size of the zones was evaluated as the number of grid cells that belong to a certain threshold of streamlines. For the case with $\alpha=6^{\circ}$ the main recirculation zone was bigger for the case with a cavity. With the increase of $\alpha$, base profile geometry tended to form a bigger recirculation zone.

Table 5. Recirculation zone comparison.

\begin{tabular}{ccccc}
\hline & Cav. A $\boldsymbol{\alpha}=\mathbf{6}^{\circ}$ & Base $\boldsymbol{\alpha}=\mathbf{6}^{\circ}$ & Cav. A $\boldsymbol{\alpha}=\mathbf{9}^{\circ}$ & Base $\boldsymbol{\alpha}=\mathbf{9}^{\circ}$ \\
\hline Recircul. zone area & $1.32 \times 10^{5}$ & $1.12 \times 10^{5}$ & $1.47 \times 10^{5}$ & $1.51 \times 10^{5}$ \\
(RZA) & $-7.94 \times 10^{6}$ & $-10^{7}$ & $-1.22 \times 10^{7}$ & $-1.28 \times 10^{7}$ \\
Total vorticity for RZA & $1.97 \times 10^{4}$ & - & $1.88 \times 10^{4}$ & - \\
Cavity zone area (CZA) & $7.52 \times 10^{5}$ & - & $1.18 \times 10^{6}$ & - \\
Total vorticity for CZA & 0.196 & 0.264 & 0.195 & 0.201 \\
Separation point, $\mathrm{x} / \mathrm{c}$ & &
\end{tabular}

The aggregated value of the vorticity in the cavity zone increase is approximately proportional to the increase in the recirculation zone. The size of this zone remains nearly the same with the $\alpha$ increase. During post-processing, a separation point was determined. The authors treated a point where the vorticity changes its sign as a point where flow detaches from the surface of a body. A strong advantage of an airfoil with a cavity is that the separation point remains the same with the increase of $\alpha$. 
To keep fluid flow analysis complete time-averaged streamlines, vorticity, and velocity distribution for $\alpha=9^{\circ}$ is presented in Figure 11. Time averaging was done for a period of fully developed flow. The solution from each step was added and then divided by the number of steps.

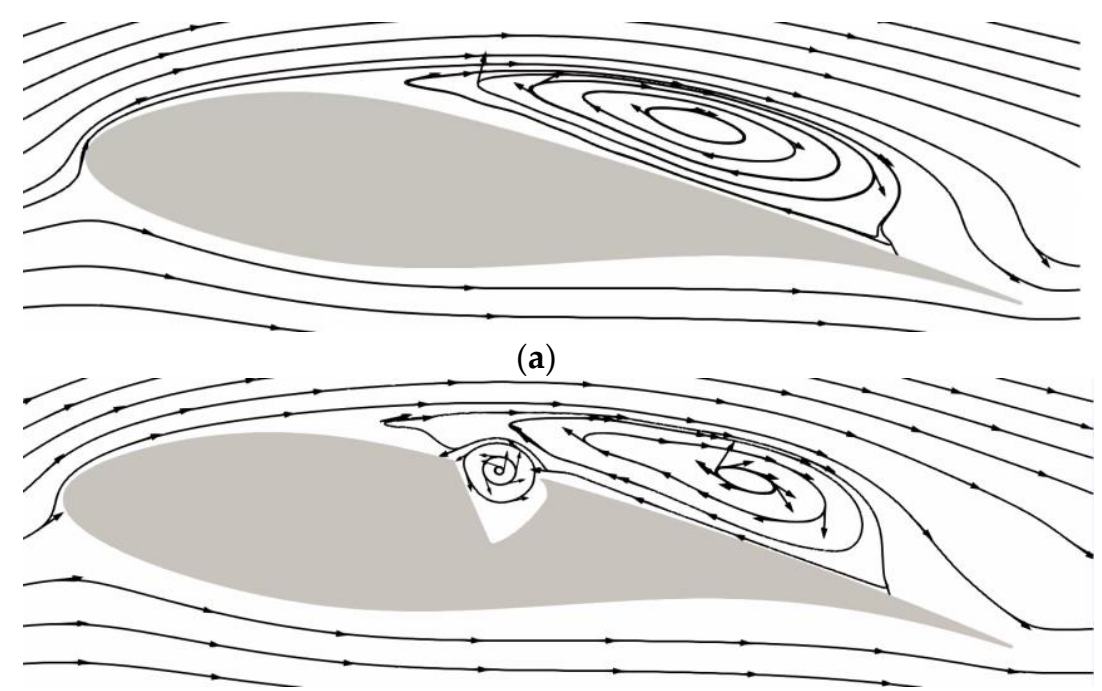

(b)

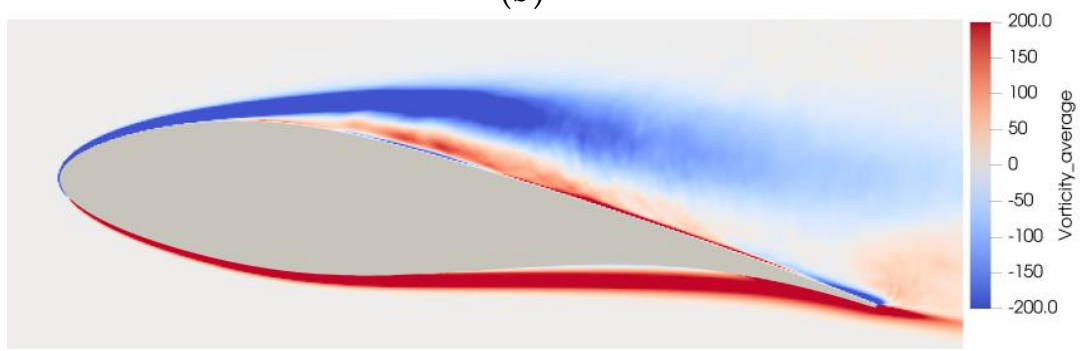

(c)

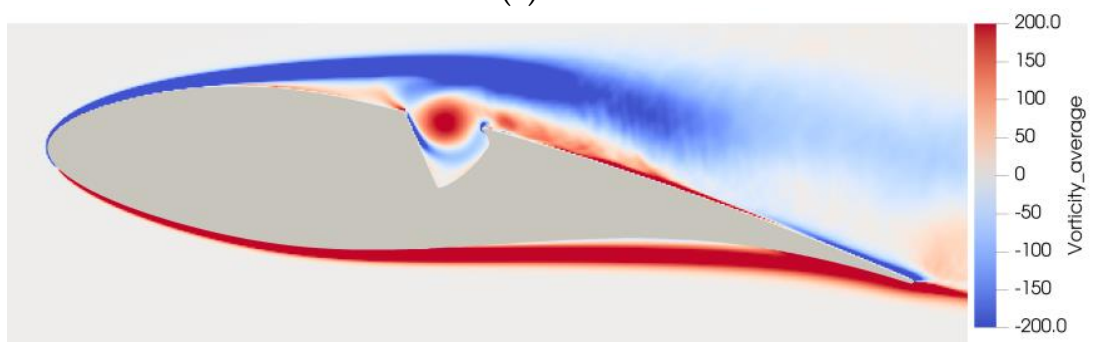

(d)

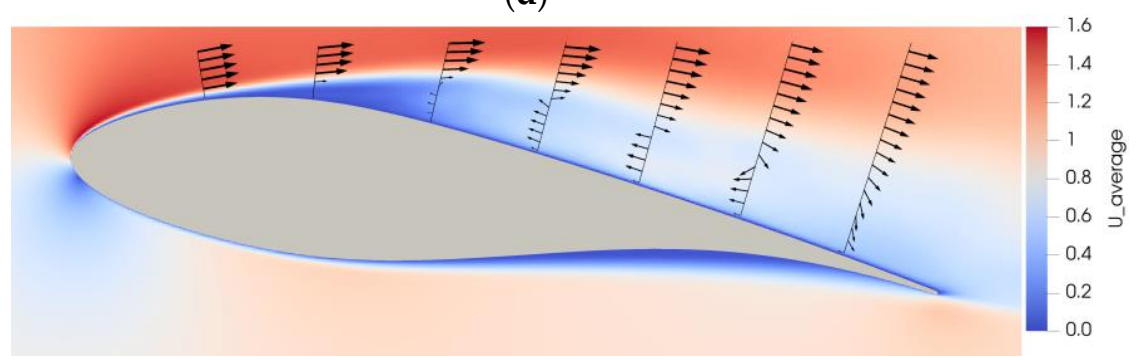

(e)

Figure 11. Cont. 


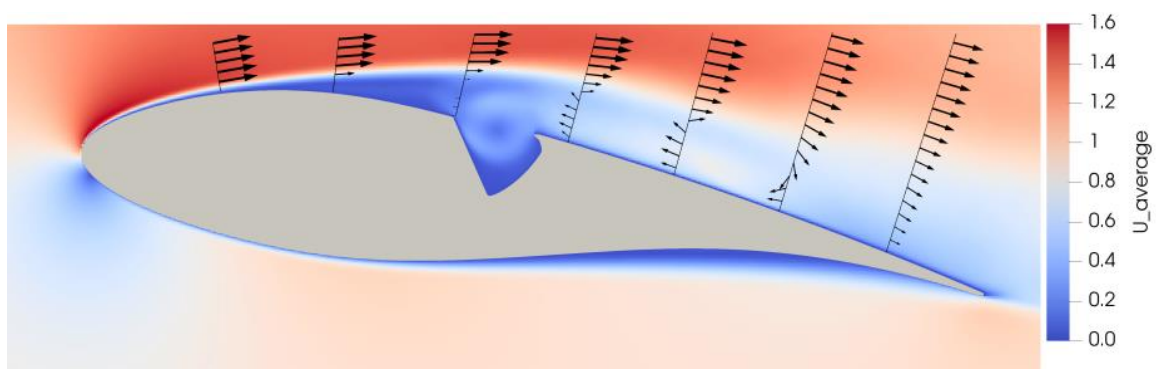

(f)

Figure 11. Time-averaged flow visualization for $\alpha=9^{\circ}$ : (a) Streamlines of standard Risø airfoil; (b) streamlines of an airfoil with cavity A; (c) vorticity contours standard Risø airfoil; (d) vorticity contours airfoil with cavity A; (e) velocity distribution for standard Risø airfoil; (f) velocity distribution for an airfoil with cavity A.

The recirculation zone is nearly the same for both angles of attack. The flow trajectory shows that the cavity vortex does not lay in the cavity itself. Its center is located at the tangent line to the upper surface. It sticks out slightly so that the main recirculation zone streamlines are deformed. The center of the zone is shifted towards the trailing edge. In the cavity, a counter-rotating swirl can be distinguished. In Figure 11d the vorticity in the cavity zone achieves a significant value.

Holding the vortex close to the top edge of the profile or delaying detachment from the leading edge is the basic phenomenon that intensifies the lift on the airfoil. According to Peskin [68], lift and drag forces are proportional to the time rate of change of the total first moment of the vorticity field $M=\left[M_{1}, M_{2}\right]^{\mathrm{T}}$ where:

$$
\begin{aligned}
M_{1}(t) & =\int_{F} y \omega(\mathbf{x}, t) d \mathbf{x}, \\
M_{2}(t) & =-\int_{F} x \omega(\mathbf{x}, t) d \mathbf{x} .
\end{aligned}
$$

Force exerted on a body that stays still or moves with a constant velocity drag and lift forces can be determined as:

$$
\begin{gathered}
F_{D}=-\rho \frac{d M_{1}}{d t}=-\rho \frac{d}{d t} \int_{F} y \omega(\mathbf{x}, t) d \mathbf{x}, \\
F_{L}=-\rho \frac{d M_{2}}{d t}=\rho \frac{d}{d t} \int_{F} x \omega(\mathbf{x}, t) d \mathbf{x},
\end{gathered}
$$

where $x, y$ are the coordinates of the aerodynamic center of a body. Therefore, the amount of lift force generated will depend on the time rate of change of the first-moment difference generated by positive and negative vorticity. This means that the lift produced of the airfoil will be strictly dependent on the actual vorticity distribution and the first moment created by vortices. Due to vorticity preservation, the total vorticity in the domain is equal to zero. It can be written that lift force is dependent on first-moment production by positive $(F P)$ and negative vorticity $(F N)$ :

$$
F_{L}=\frac{d}{d t} \rho \int_{F P} x \omega(\mathbf{x}, t) d \mathbf{x}-\frac{d}{d t} \rho \int_{F N} x \omega(\mathbf{x}, t) d \mathbf{x} .
$$

The analysis indicates that the correlation of vorticity field evolution, Figure 12, can explain the additional lift generation for an airfoil with a cavity. 


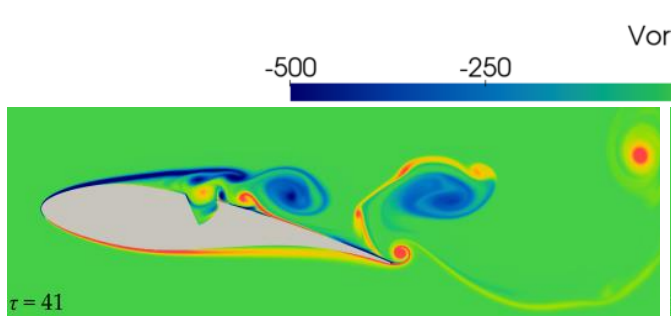

(a)

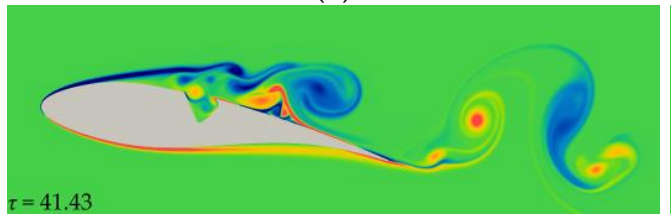

(c)

\section{O}

0
500

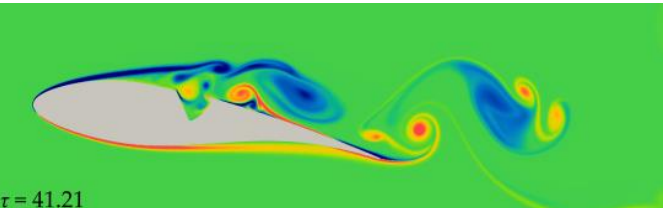

(b)

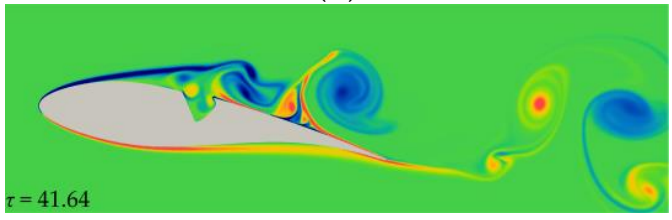

(d)

Figure 12. Evolution of vorticity for an airfoil with cavity $A, \alpha=9^{\circ}$, sequence of images at (a) $\tau=41$, (b) $\tau=41.21$, (c) $\tau=41.43$, (d) $\tau=41.64$.

From Equation (31) it can be stated that an increase in lift force is observed if the vortex of positive vorticity is advected away from the body and vortex with negative vorticity is held close to the body. It was observed that due to the presence of the cavity, the negative vortex structure stays longer on the top surface of the profile.

The wake with negative vorticity in a standard airfoil leaves the surroundings of the airfoil earlier. This results in a lower difference between the rate of change of the first moment caused by positive and negative vorticity and lift force generation in general.

\section{Experimental Method}

In this paper, experimental data obtained in a water tunnel will be presented. This section gives a brief description of the experimental facility.

The experiments were conducted in the 2436 Rolling Hill Research Corporation Flow Visualization Water Tunnel [69]. This is a closed-circuit facility being operated as a continuous flow channel. The test section is nominally $24^{\prime \prime}$ wide, 36 " high, and 72 " long. It is constructed principally of tempered glass to permit maximum viewing of the model. The test section and discharge plenum are configured to allow simultaneous viewing of a model from all sides. The test section flow velocity can vary from 0 up to $1 \mathrm{ft} / \mathrm{s}$.

The three airfoils presented in Section 5 were manufactured out of transparent UV curing resin, have a chord length of $c=300 \mathrm{~mm}$, and a sharpened trailing edge. To minimize end effects and create quasi-two-dimensional flow over the airfoil, the airfoil is bounded at the ends by two transparent Plexiglas end plates-presented for the airfoil with cavity A at Figure 13. The dimensions of the plates were $500 \times 350 \mathrm{~mm}$ and had a thickness of $4 \mathrm{~mm}$. To prevent flow separation, the upstream edges of the endplates were rounded. The airfoil was mounted in the middle of the endplates.

The formation of vortex structures on the airfoil as well as the effect of the cavity on the behavior of these vortex structures was investigated using dye visualization. Fluorescein $\left(\mathrm{C}_{20} \mathrm{H}_{12} \mathrm{O}_{5}\right)$ was used as a dye in the research.

Each of the tested profiles (without a cavity, with cavity A and with a cavity B-Figure 7) were placed vertically in the test section of the water tunnel. In each case, during the visualization tests, the direction of water flow was from left to right, so the stream was flowing on the leading edge of the profile.

For the standard Risø airfoil, the dye injection holes were located on the top surface at a distance of $0.1 c$ (hole 1 ) and $0.5 c$ (hole 2)-Figure 14a. For the airfoil with cavity A, the dye injection holes were located at a distance of $0.1 c$ (hole 1 ) and $0.6 c$ (hole 2), as shown in Figure $14 \mathrm{~b}$. For the airfoil with cavity B-at a distance of $0.1 c$ (hole 1 ) and $0.7 c$ (hole 2), as shown in Figure 14c. In each case, the dye was discharged simultaneously from both sides. The internal diameter of the holes through which the dye was led out was $0.5 \mathrm{~mm}$ for each 
analyzed profile shape. An infusion pump was used to precisely dose small amounts of dye. One should be careful of excessive dye flow rate because this can disturb the formation of the vortex structure that is to be observed. For each tested profile visualizations were carried out for four values of the Reynolds number (based on the airfoil chord: $R e=10^{4}$; $\left.2 \times 10^{4} ; 3 \times 10^{4} ; 4 \times 10^{4}\right)$ and five values of the angle of attack $\left(\alpha=0^{\circ} ; 3^{\circ} ; 6^{\circ} ; 9^{\circ} ; 12^{\circ}\right)$ within each Reynolds number.

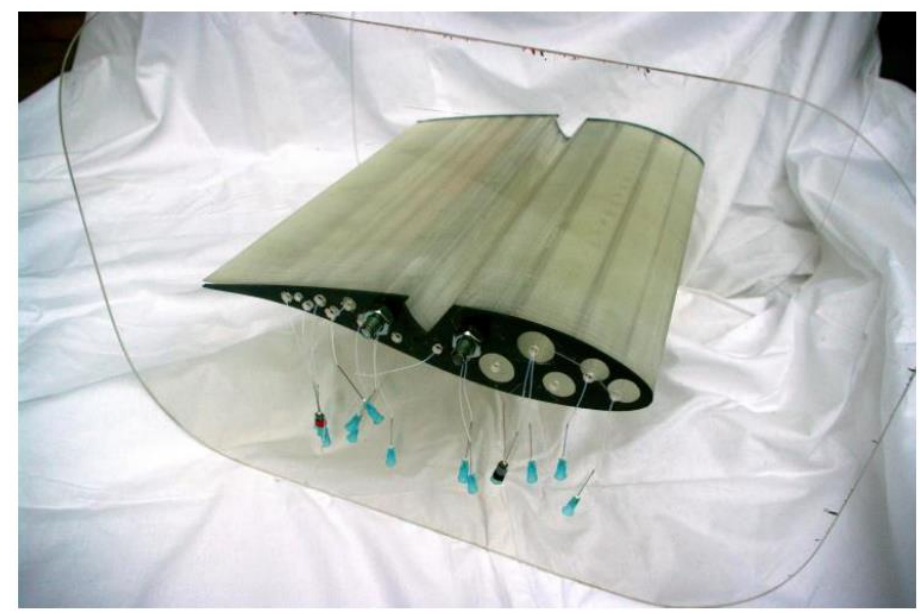

Figure 13. Risø airfoil with cavity A-model prepared for visualization tests.

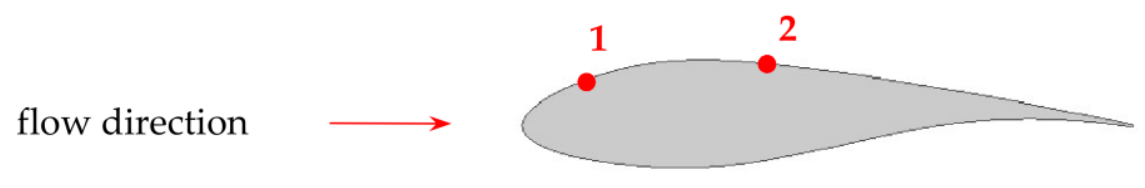

(a)

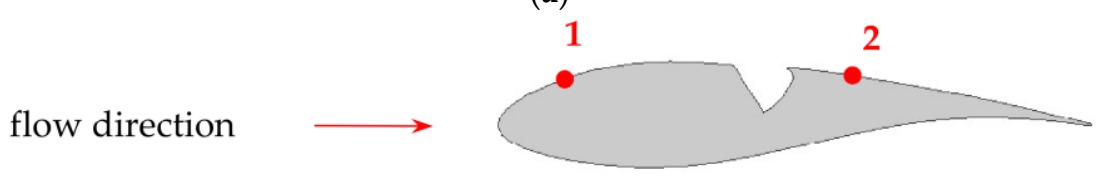

(b)

1

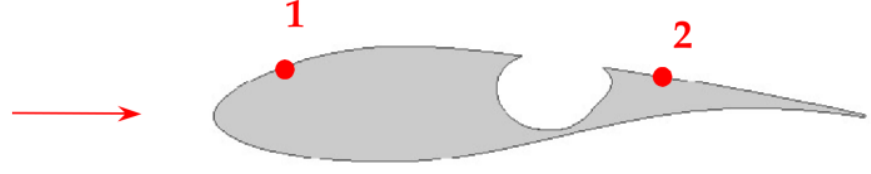

flow direction

(c)

Figure 14. Location of the dye injection holes: (a) standard Risø airfoil; (b) airfoil with cavity A; (c) airfoil with cavity B.

\section{Experimental Results}

In this section the results of the flow visualization in the water tunnel for $R e=2 \times 10^{4}$ are presented. Images are shown for a nondimensional time calculated as $\tau=t \cdot u_{\infty} / c$, where $t$-time after which the image was obtained, $u_{\infty}$-velocity in the water tunnel corresponding to $\operatorname{Re}=2 \times 10^{4}, c$ - the airfoil chord.

Exemplary images of flow around the standard Risø airfoil for $R e=2 \times 10^{4}$ are presented in Figures 15 and 16. For the angle of attack $\alpha=6^{\circ}$, a clear vortex structure was observed on the upper surface of the profile. The dye from hole 2 was transported both towards the leading edge and taken in the direction of the main flow. An increase in the angle of attack to $\alpha=9^{\circ}$ intensified the processes already observed for $\alpha=6^{\circ}$. The detachment point moved towards the leading edge with the increase of the angle of attack. 


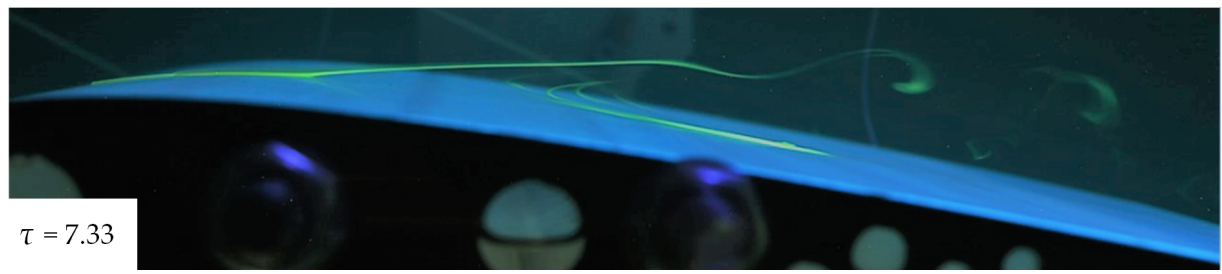

(a)

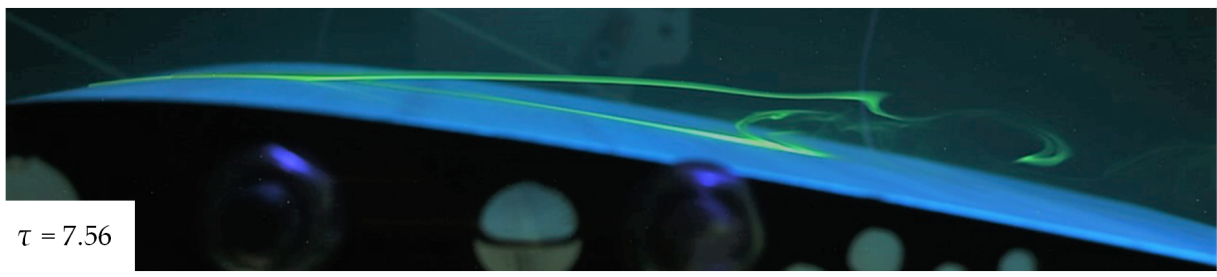

(b)

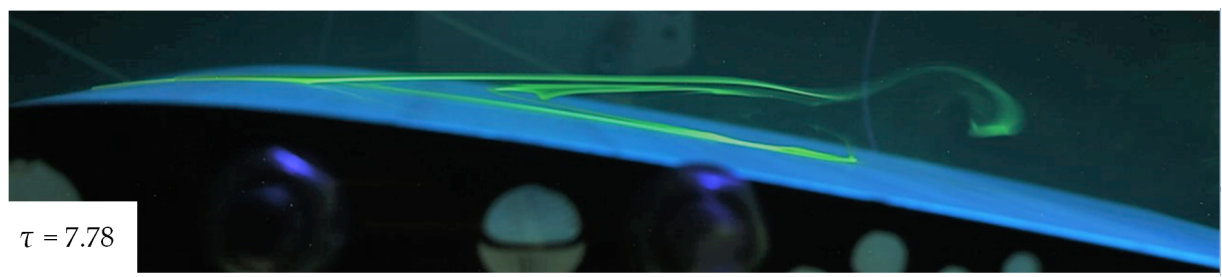

(c)

Figure 15. Flow images for standard Ris $\varnothing$ at $R e=2 \times 10^{4}, \alpha=6^{\circ}:(\mathbf{a}) \tau=7.33$; (b) $\tau=7.56$; (c) $\tau=7.78$.

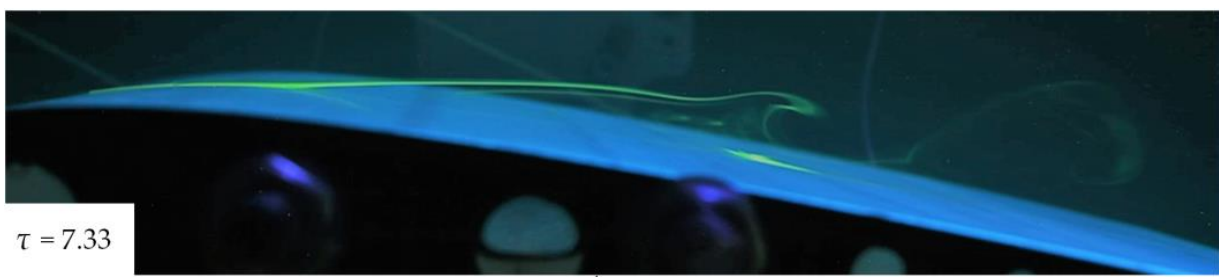

(a)

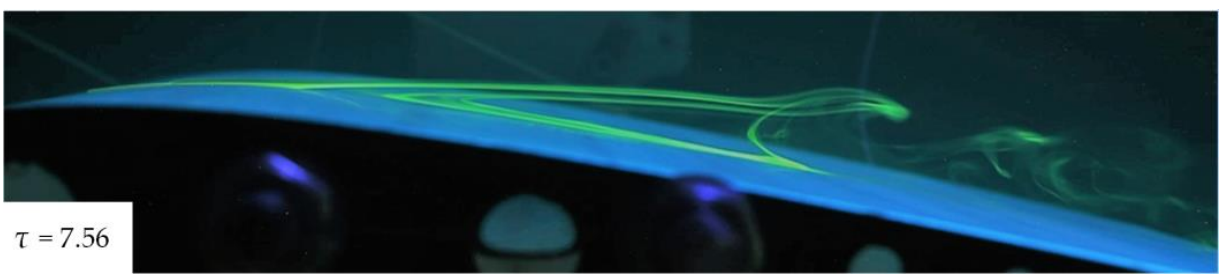

(b)

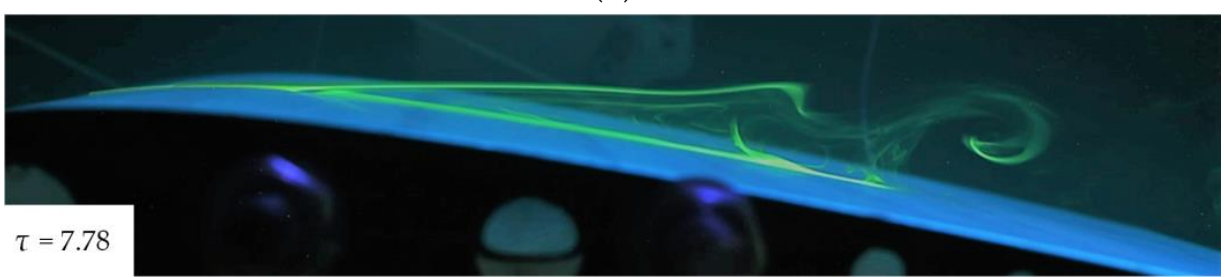

(c)

Figure 16. Flow images for standard Risø at $R e=2 \times 10^{4}, \alpha=9^{\circ}:(\mathbf{a}) \tau=7.33$; (b) $\tau=7.56$; (c) $\tau=7.78$.

Images of the flow around the airfoil with cavity A (sharp cavity) and $R e=2 \times 10^{4}$ are presented in Figures 17 and 18. For $\alpha=6^{\circ}$ the dye given from hole 2 flowed into the cavity. It did not flow through the top of the cavity, it headed to the front wall of the cavity. Here, it split, creating two dye threads. One of them flowed up, the second one flowed down. The part of the dye that flowed to the bottom of the cavity gives the outline of a structure that rotates opposite to the streamwise direction. It is difficult to judge the direction of rotation. On the surface of the airfoil behind the cavity, strong dye dissipation was visible. 


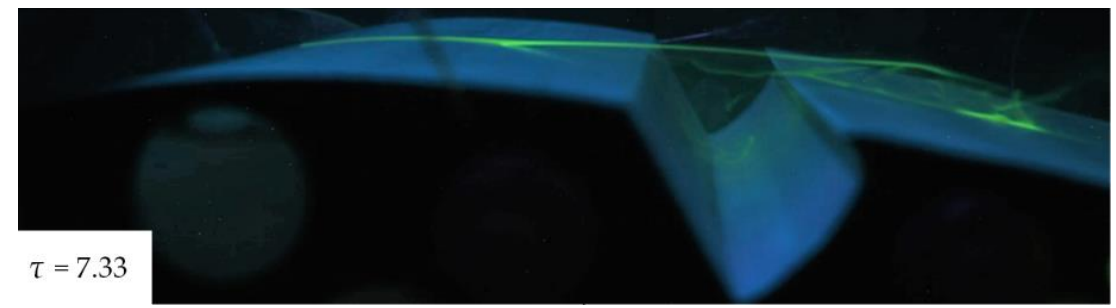

(a)

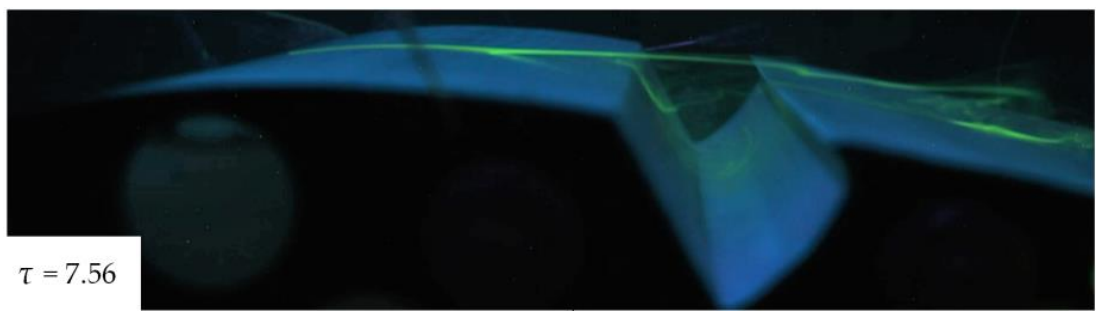

(b)

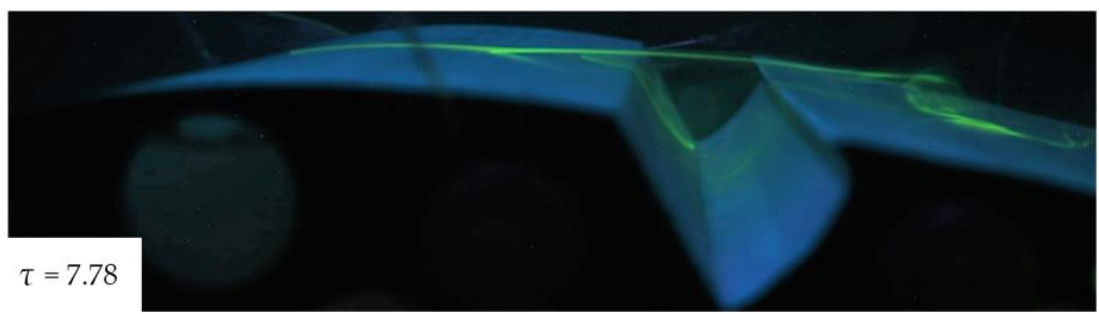

(c)

Figure 17. Flow images for cavity A at $\operatorname{Re}=2 \times 10^{4}, \alpha=6^{\circ}:$ (a) $\tau=7.33$; (b) $\tau=7.56$; (c) $\tau=7.78$.

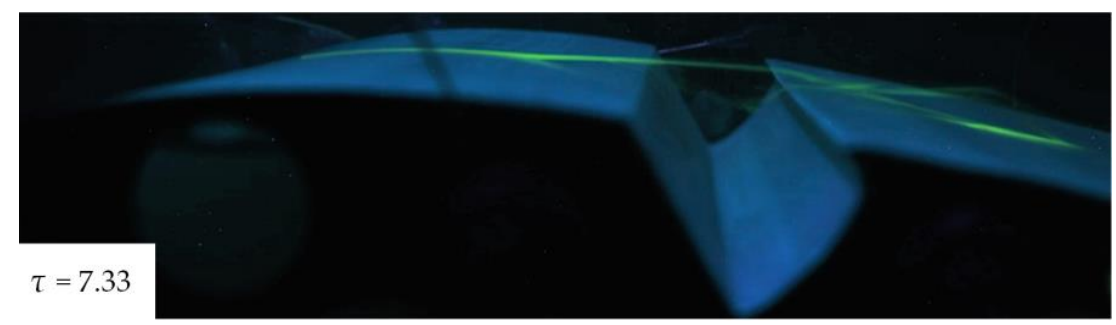

(a)

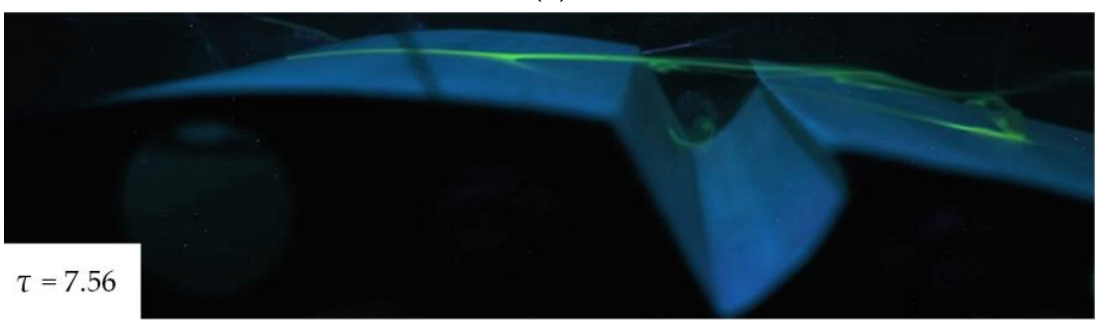

(b)

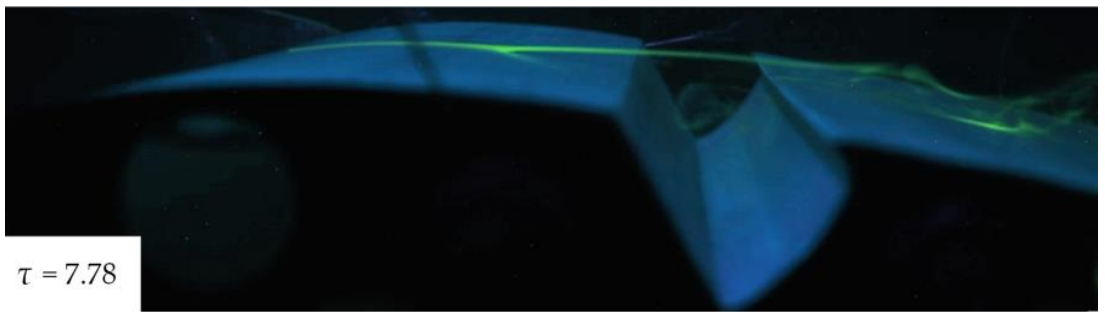

(c)

Figure 18. Flow images for cavity $\mathrm{A}$ at $\operatorname{Re}=2 \times 10^{4}, \alpha=9^{\circ}:(\mathbf{a}) \tau=7.33$; (b) $\tau=7.56$; (c) $\tau=7.78$. 
For $\alpha=9^{\circ}$ strong eddies are visible on the upper surface of the profile behind the cavity. A small amount of dye flows into the cavity and forms a structure that rotates in the direction opposite to the streamwise direction.

Images of the flow around the airfoil with cavity B (big cavity) and $R e=2 \times 10^{4}$ are presented in Figures 19 and 20. For $\alpha=6^{\circ}$ the dye from hole 2 moves towards the cavity and flows down from its rear edge. The dye flows towards the upper edge of the front wall of the cavity. This separates the dye into 2 threads. One of them flows downwards and forms a large vortex structure that rotates against the main flow. The second one flows on the front part of the upper surface of the profile, and here it creeps under the dye given from hole 1.

The increase of the angle of attack to $\alpha=9^{\circ}$ causes that the amount of dye flowing into the cavity is much smaller than it was for $\alpha=6^{\circ}$. This is due to the entrainment of a large part of the dye by the stream visualized by the dye given from hole 1. Additionally, for this case, the dye is much more dispersed both in the cavity and behind it-on the upper surface of the airfoil than it was observed for $\alpha=6^{\circ}$.

For the airfoil with cavity B, one can observe the boundary layer eruptions on the upper surface of the profile behind the cavity for each angle of attack.

The results of the qualitative experimental tests are in good agreement with the results of the numerical calculations. Vortex structures are formed similarly for both cases.

The location of the separation point and the size of the detachment area above the top surface of the profile, obtained in the numerical results, agree with the images obtained by visualizing the flow in a water tunnel.

Moreover, the relationship between the increase in the intensity of the liquid spinning inside the cavity and the increase in the angle of attack of the profile is also consistent.

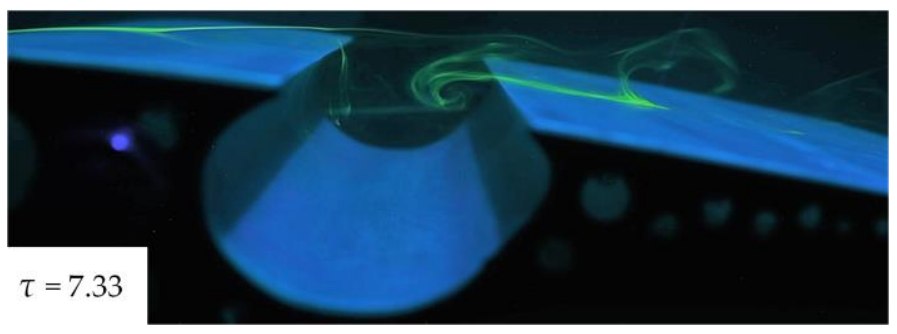

(a)

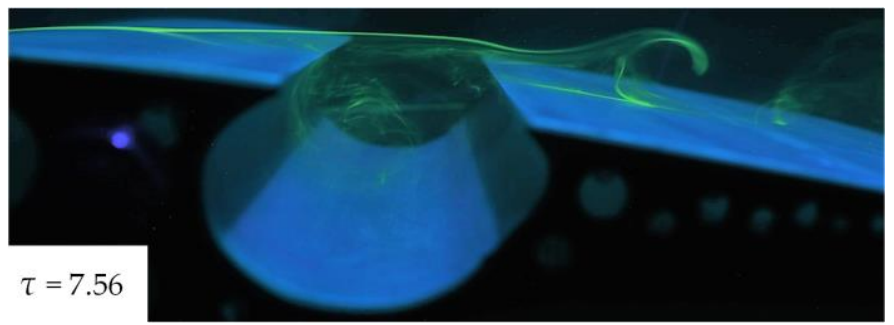

(b)

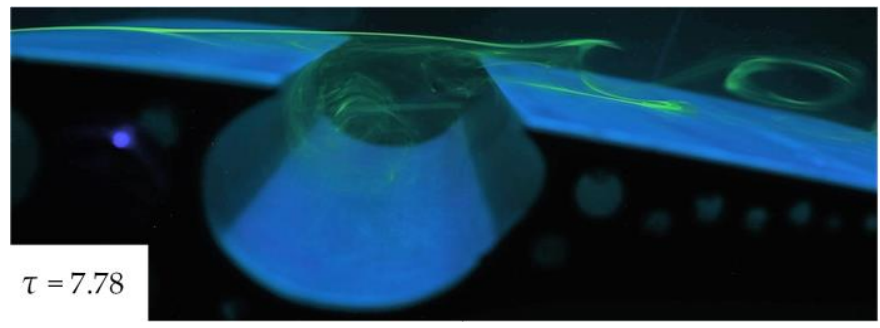

(c)

Figure 19. Flow images for cavity $B$ at $R e=2 \times 10^{4}, \alpha=6^{\circ}:$ (a) $\tau=7.33 ;$ (b) $\tau=7.56$; (c) $\tau=7.78$. 


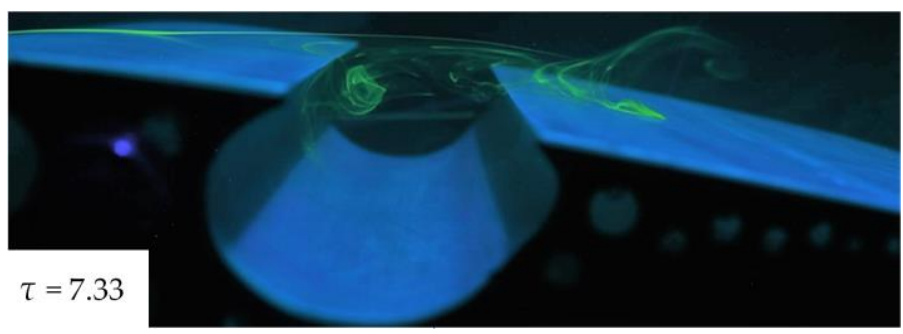

(a)

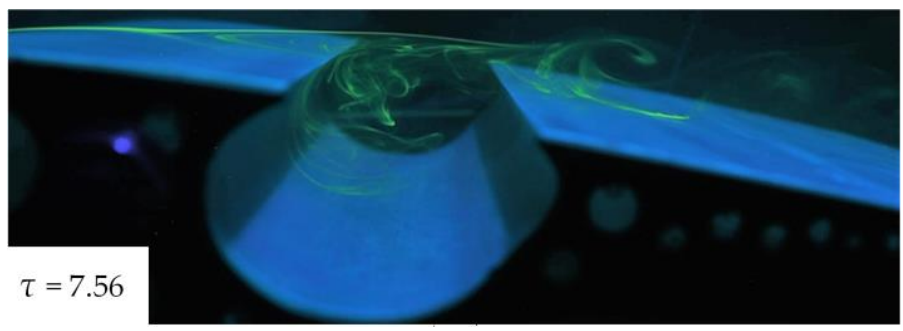

(b)

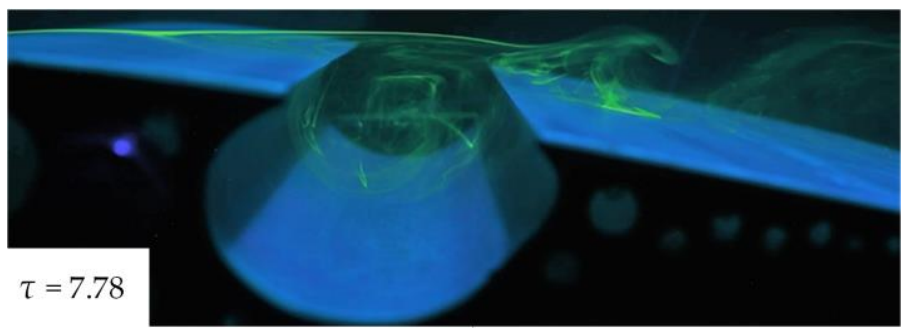

(c)

Figure 20. Flow images for cavity $B$ at $R e=2 \times 10^{4}, \alpha=9^{\circ}:$ (a) $\tau=7.33$; (b) $\tau=7.56$; (c) $\tau=7.78$.

The images obtained by visualization, showing the evolution of the flow over all the analyzed aerodynamic profiles, are very important for the authors of this article, as they confirm the correctness of numerical calculations in which a flow of similar nature and behavior was observed.

\section{Conclusions}

The high-order penalized vortex in cell method applied to solve the flow past an airfoil with vortex trapping cavity was presented. The numerical implementation of the method and high-order schemes incorporated into the algorithm was discussed. Poisson, stream-velocity, advection, and diffusion equations were solved. The derivation, finite difference formulation, Lagrangian particle remeshing procedure, and accuracy tests were shown.

Simulations and experimental results of the flow over standard Risø airfoil as well as over Risø airfoils equipped with two cases of vortex trapping cavities (cavity A and cavity B) were presented. This paper presents the results of the research carried out for the Reynolds number (based on the airfoil chord) $R e=2 \times 10^{4}$ and selected values of the angle of attack.

For all investigated cases, cavity A on the upper surface of an airfoil led to an increase of lift coefficient due to additional circulation around a profile. The presence of the cavity deteriorated the $C_{L} / C_{D}$ ratio for $\alpha=3^{\circ}$ and $\alpha=6^{\circ}$. For $\alpha=9^{\circ}$, cavity A outperformed the smooth profile. The $C_{L} / C_{D}$ ratio was higher for an airfoil with cavity $A$ than for the standard Risø airfoil. The drag coefficient was higher for a profile with a cavity in respect to the smooth profile in all cases and it increased with the increase of the angle of attack. Additionally, it should be noticed that the optimal angle of attack moved toward higher values. 
The results of qualitative experimental tests are in good agreement with the results of numerical calculations. Similar vortex structures forming in a similar way were observed. Images obtained by visualization, showing the evolution of the flow over all the analyzed aerodynamic profiles are very important for the authors. They confirm the correctness of numerical calculations in which a flow of similar nature and behavior was observed.

Author Contributions: Conceptualization, D.B. and H.K.; methodology, H.K., D.B., K.S.; software, D.B.; validation, K.S., D.B.; formal analysis, H.K.; investigation, D.B.; data curation, K.S.; writingoriginal draft preparation, D.B., K.S. and H.K.; writing—review and editing, H.K.; visualization, K.S.; supervision, H.K.; project administration, D.B.; All authors have read and agreed to the published version of the manuscript.

Funding: This research received no external funding.

Acknowledgments: Calculations have been made using resources provided by Wroclaw Centre for Networking and Supercomputing (http:/ / wcss.pl, accessed on 29 October 2021), grant No. 444/2021.

Conflicts of Interest: The authors declare no conflict of interest.

\section{Nomenclature}

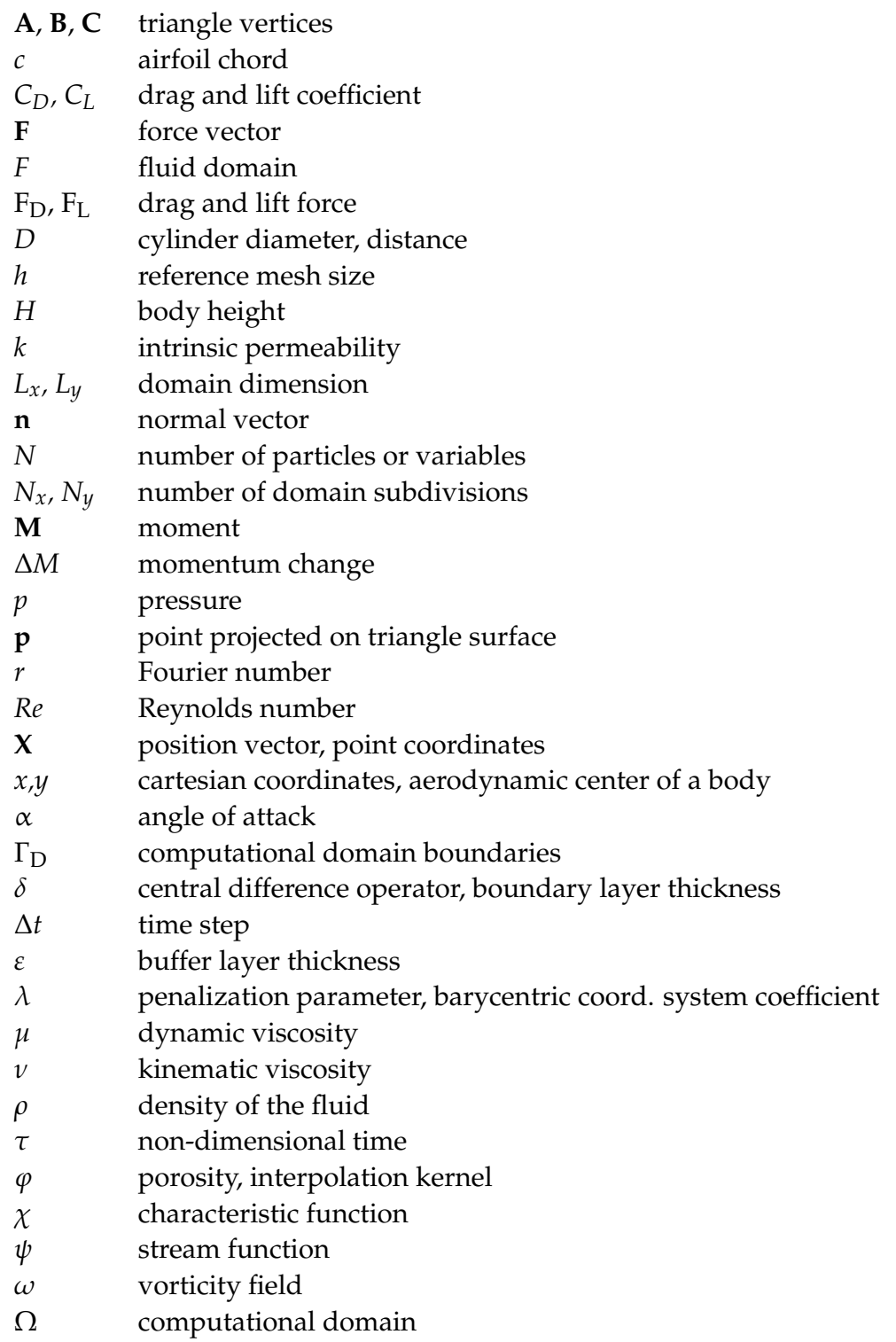




$\begin{array}{ll}\text { Abbreviations } & \\ \text { CZA } & \text { cavity zone area } \\ \text { CycRed } & \text { cyclic reduction } \\ \text { CFL } & \text { Courant-Friedrichs-Lewy } \\ \text { GCI } & \text { grid convergence index } \\ \text { GMRES } & \text { generalized minimal residual method } \\ \text { LHS } & \text { left-hand side } \\ \text { LE } & \text { leading edge } \\ \text { MPI } & \text { message passing interface } \\ \text { PFMG } & \text { parallel semicoarsening multigrid } \\ \text { PIV } & \text { particle image velocimetry } \\ \text { RHS } & \text { right-hand side } \\ \text { RZA } & \text { recirculation zone area } \\ \text { SMG } & \text { semicoarsening multigrid } \\ \text { TE } & \text { trailing edge } \\ \text { TSR } & \text { tip speed ratio } \\ \text { TVC } & \text { trapped vortex cavity } \\ \text { UV } & \text { ultraviolet light } \\ \text { VIC } & \text { vortex in cell }\end{array}$

\section{References}

1. Stępień, M.; Kulak, M.; Jóźwik, K. "Fast Track” Analysis of Small Wind Turbine Blade Performance. Energies 2020, $13,5767$. [CrossRef]

2. Verma, D.R.; Katkade, S.D. Horizontal Axis Water Turbine: Generation and Optimization of Green Energy. Int. J. Appl. Eng. Res. 2018, 13, 5767. [CrossRef]

3. Battisti, L.; Brighenti, A.; Benini, E.; Castelli, M.R. Analysis of Different Blade Architectures on small VAWT Performance. J. Phys. Conf. Ser. 2016, 753, 062009. [CrossRef]

4. Fuglsang, P.; Bak, C. Development of the Risø Wind Turbine Airfoils. Wind Energy 2004, 7, 145-162. [CrossRef]

5. Yeung, W.W.H. Lift enhancement on unconventional airfoils. J. Mek. 2006, 22, 17-25.

6. Hau, E.; Renouard, H.V. Wind Turbines—Fundamentals, Technologies, Application, Economics; Springer: Berlin/Heidelberg, Germany, 2006.

7. Bak, C. Sensitivity of Key Parameters in Aerodynamic Wind Turbine Rotor Design on Power and Energy Performance. J. Phys. Conf. Ser. 2007, 75, 012008. [CrossRef]

8. Yen, J.; Ahmed, N.A. Parametric study of dynamic stall flow field with synthetic jet actuation. J. Fluids Eng. 2012, 134, 071106. [CrossRef]

9. Yen, J.; Ahmed, N.A. Corrigendum to Enhancing vertical axis wind turbine by dynamic stall control using synthetic jets. J. Wind Eng. Ind. Aerodyn. 2013, 114, 12-17. [CrossRef]

10. Ma, X.; Schröder, A. Visualization of separated shear layer streaks generated by micro vortex generators based on tomographic PIV. J. Vis. 2018, 21, 185-190. [CrossRef]

11. Al-Jaburi, K.; Feszty, D. Passive flow control of dynamic stall via surface-based trapped vortex generators. J. Am. Helicopter Soc. 2018, 63, 1-14. [CrossRef]

12. Nematollahi, O.; Nili-Ahmadabadi, M.; Seo, H.; Kim, K.C. Effect of acicular vortex generators on the aerodynamic features of a slender delta wing. J. Aerosp. Sci. Technol. 2019, 86, 327-340. [CrossRef]

13. Moshfeghi, M.; Shams, S.; Hur, N. Aerodynamic performance enhancement analysis of horizontal axis wind turbines using a passive flow control method via split blade. J. Wind Eng. Ind. Aerodyn. 2017, 167, 148-159. [CrossRef]

14. Nafar-Sefiddashti, M.; Nili-Ahmadabadi, M.; Rizi, B.S.; Pourhoseini, J. Visualization of flow over a thick airfoil with circularcross-section riblets at low Reynolds numbers. J. Vis. 2019, 22, 877-888. [CrossRef]

15. Luo, D.; Huang, D.; Sun, X. Passive flow control of a stalled airfoil using a microcylinder. J. Wind Eng. Ind. Aerodyn. 2017, 170, 256-273. [CrossRef]

16. De Gregorio, F.; Fraioli, G. Flow control on a high thickness airfoil by a trapped vortex cavity. In Proceedings of the 14th International Symposium on Applications of Laser Techniques to Fluid Mechanics, Lisbon, Portugal, 7-10 July 2008.

17. Olsman, W.F.J.; Colonius, T. Numerical simulation of flow over an airfoil with a cavity. IAA J. 2011, 49, 143-149. [CrossRef]

18. Chernyshenko, S.I.; Galletti, B.; Iollo, A.; Zannetti, L. Trapped vortices and a favorable pressure gradient. J. Fluid Mech. 2003, 482, 235-255. [CrossRef]

19. Baranov, P.; Guvernyuk, S.; Zubin, M.; Isaev, S. Numerical and physical modeling of the circulation flow in a vortex cell in the wall of a rectilinear channel. Fluid Dyn. 2000, 35, 663-673. [CrossRef]

20. Fertis, D.G. New airfoil-design concept with improved aerodynamic characteristics. J. Aerosp. Eng. 1994, 7, 328-339. [CrossRef] 
21. Rockwell, D.; Naudascher, E. Self-sustaining oscillations of flow past cavities. J. Fluids Eng. 1978, 100, 152-165. [CrossRef]

22. Rockwell, D.; Naudascher, E. Self-sustained oscillations of impinging free shear layers. Annu. Rev. Fluid Mech. 1979, 11, 67-94. [CrossRef]

23. Olsman, W.F.J.; Willems, J.F.H.; Hirschberg, A.; Colonius, T.; Trieling, R.R. Flow around a NACA0018 airfoil with a cavity and its dynamical response to acoustic forcing. Exp. Fluids 2011, 51, 493-509. [CrossRef]

24. Yeung, W.W.H. Vortex trapping on a surface with an indentation and corrugations. Math. Comput. Simul. 2009, $79,3243-3257$. [CrossRef]

25. Timmer, W.A. Two-dimensional low-Reynolds number wind tunnel results for airfoil NACA 0018. Wind Eng. 2008, 32, 525-537. [CrossRef]

26. Vuddagiri, A.; Samad, A. Vortex Trapping by Different Cavities on an Airfoil. Wind Eng. 2013, 37, 469-482. [CrossRef]

27. Vuddagiri, A.; Halder, P.; Samad, A.; Chaudhuri, A. Flow analysis of airfoil having different cavities on its suction surface. Comput. Fluid Dyn. 2016, 16, 67-77. [CrossRef]

28. Fatehi, M.; Nili-Ahmadabadi, M.; Nematollahi, O.; Minaiean, A.; Kim, K.C. Aerodynamic performance improvement of wind turbine blade by cavity shape optimization. Renew. Energy 2019, 132, 773-785. [CrossRef]

29. Nili-Ahmadabadi, M.; Nematollahi, O.; Fatehi, M.; Cho, D.S.; Kim, K.C. Evaluation of aerodynamic performance enhancement of Risø_B1 airfoil with an optimized cavity by PIV measurement. J. Vis. 2020, 23, 591-603. [CrossRef]

30. Djojodihardjo, H. Progress and development of Coanda jet and vortex cell for aerodynamic surface circulation control—An overview. SIJ Trans. Adv. Space Res. Earth Explor. 2013, 1, 32-42. [CrossRef]

31. Lasagna, D.; Donelli, R.; De Gregorio, F.; Iuso, G. Effects of a trapped vortex cell on a thick wing airfoil. Exp. Fluids 2011, 51. [CrossRef]

32. Cottet, G.-H.; Koumoutsakos, P.D. Vortex Methods: Theory and Practice; Cambridge University Press: Cambridge, UK, 2000 [CrossRef]

33. Mimeau, C.; Mortazavi, I.A. Review of Vortex Methods and Their Applications: From Creation to Recent Advances. Fluids 2021, 6, 68. [CrossRef]

34. Kosior, A.; Kudela, H. Parallel computations on GPU in 3D using the vortex particle method. Comput. Fluids 2013, 80, 423-428. [CrossRef]

35. van Rees, W.M.; Leonard, A.; Pullin, D.; Koumoutsakos, P. A comparison of vortex and pseudo-spectral methods for the simulation of periodic vortical flows at high Reynolds numbers. J. Comput. Phys. 2011, 230, 2794-2805. [CrossRef]

36. Cocle, R.; Dufresne, L.; Winckelmans, G. Investigation of multiscale subgrid scale models for LES of instabilities and turbulence in wake vortex systems. In Complex Effects in Large Eddy Simulations, Lecture Notes in Computational Science and Engineering (LNCSE); Springer: Berlin/Heidelberg, Germany, 2007; Volume 56. [CrossRef]

37. Rossinelli, D.; Hejazialhosseini, B.; van Rees, W.M.; Gazzola, M.; Bergdorf, M.; Koumoutsakos, P. MRAG-I2D: Multi-resolution adapted grids for remeshed vortex methods on multicore architectures. J. Comput. Phys. 2015, 288, 1-18. [CrossRef]

38. Kudela, H.; Kozlowski, T. Vortex-in-cell method for exterior problems. J. Theor. Appl. Mech. 2009, 47, 779-796.

39. Błoński, D. Implementacja metody „Wir w Komórce” w środowisku wieloprocesorowym dla zagadnienia przepływu w komorze $\mathrm{z}$ ruchomą ścianką. Zesz. Energetyczne 2019, 6, 27-44.

40. Błoński, D. Realizacja metody czastek wirowych w środowisku wieloprocesowym z użyciem schematów różnicowych wysokiego rzędu. Zesz. Energetyczne 2018, 5, 25-40.

41. Morency, F.; Beaugendre, H.; Gallizio, F. Aerodynamic force evaluation for ice shedding phenomenon using vortex in cell scheme, penalisation and level set approaches. Int. J. Comput. Fluid Dyn. 2012, 26, 435-450. [CrossRef]

42. Angot, P.; Bruneau, C.-H.; Fabrie, P. A penalization method to take into account obstacles in incompressible viscous flows. Numer. Math. 1999, 81, 497-520. [CrossRef]

43. Bost, C.; Cottet, G.-H.; Maitre, E. Numerical analysis of a penalization method for the three-dimensional motion of a rigid body in an incompressible viscous fluid. arXiv 2009, arXiv:0901.1958.

44. Baker, A.H.; Falgout, R.D.; Gamblin, T.; Kolev, T.V.; Schulz, M.; Yang, U.M. Scaling algebraic multigrid solvers: On the road to exascale. In Competence in High Performance Computing; Springer: Berlin/Heidelberg, Germany, 2010; pp. 215-226. [CrossRef]

45. Wang, Y.; Zhang, J. Sixth order compact scheme combined with multigrid method and extrapolation technique for 2D Poisson equation. J. Comput. Phys. 2009, 228, 137-146. [CrossRef]

46. Zhang, J. Multigrid method and fourth order compact difference scheme for 2D Poisson equation with unequal mesh-size discretization. J. Comput. Phys. 2002, 179, 170-179. [CrossRef]

47. Zhao, J. Compact finite difference methods for high order integro-differential equations. Appl. Math. Comput. 2013, 221, 66-78. [CrossRef]

48. Fishelov, D.; Ben-Artzi, M.; Croisille, J.-P. Recent Advances in the Study of a Fourth-Order Compact Scheme for the OneDimensional Biharmonic Equation. J. Sci. Comput. 2012, 53, 55-79. [CrossRef]

49. Mimeau, C.; Gallizio, F.; Cottet, G.-H.; Mortazavi, I. Vortex penalization method for bluff body flows. Int. J. Numer. Methods Fluids 2015, 79, 55-83. [CrossRef]

50. Rossinelli, D.; Bergdorf, M.; Cottet, G.-H.; Koumoutsakos, P. GPU accelerated simulations of bluff body flows using vortex particle methods. J. Comput. Phys. 2010, 229, 3316-3333. [CrossRef] 
51. point2trimesh( ) —Distance Between Point and Triangulated Surface, Version 1.0.0.0 by Daniel Frisch. Available online: https://de. mathworks.com/matlabcentral/fileexchange/52882-point2trimesh-distance-between-point-and-triangulated-surface (accessed on 15 August 2021).

52. Ericson, C. Real-Time Collision Detection Christer Ericson; Springer: Berlin/Heidelberg, Germany, 2005; pp. 46-48. [CrossRef]

53. Coquerelle, M.; Cottet, G.-H. A vortex level set method for the two-way coupling of an incompressible fluid with colliding rigid bodies. J. Comput. Phys. 2008, 227, 9121-9137. [CrossRef]

54. Sagredo, J.T.B. Z-splines: Moment conserving cardinal spline interpolation of compact support for arbitrarily spaced data. In Seminar für Angewandte Mathematik; Eidgenössische Technische Hochschule: Zürich, Switzerland, 2003.

55. Cottet, G.-H.; Etancelin, J.-M.; Perignon, F.; Picard, C. High order semi-Lagrangian particle methods for transport equations: Numerical analysis and implementation issues. ESAIM M2AN 2014, 48, 1029-1060. [CrossRef]

56. Magni, A.; Cottet, G.-H. Accurate, non-oscillatory, remeshing schemes for particle methods. J. Comput. Phys. 2012, 231, 152-172. [CrossRef]

57. Liao, W.; Zhu, J.; Khaliq, A.Q.M. An efficient high-order algorithm for solving systems of reaction-diffusion equations. Numer. Methods Partial. Differ. Equ. 2002, 18, 340-354. [CrossRef]

58. Liao, W. A Compact High-Order Finite Difference Method for Unsteady Convection-Diffusion Equation. Int. J. Comput. Methods Eng. Sci. Mech. 2012, 13, 135-145. [CrossRef]

59. Beaugendre, H.; Morency, F.; Gallizio, F.; Laurens, S. Computation of Ice Shedding Trajectories Using Cartesian Grids, Penalization, and Level Sets. Hindawi Publ. Corp. Model. Simul. Eng. 2011. [CrossRef]

60. Noca, F.; Shiels, D.; Jeon, D. A comparison of methods for evaluating time-dependent fluid dynamic forces on bodies, using only velocity fields and their derivatives. J. Fluids Struct. 1999, 13, 551-578. [CrossRef]

61. Gillis, T.; Marichal, Y.; Winckelmans, G.; Chatelain, P. A 2D immersed interface Vortex Particle-Mesh method. J. Comput. Phys. 2019, 394, 700-718. [CrossRef]

62. Beatson, R.; Greengard, L. Wavelets, multilevel methods, and elliptic PDEs. In A Short Course on Fast Multipole Methods; Ainsworth, M., Levesley, J., Marletta, M., Light, W.A., Eds.; Oxford University Press: Oxford, UK, 1997; pp. 1-37.

63. Lee, S.-J.; Lee, J.-H.; Suh, J.-C. Numerical investigation on vortex shedding from a hydrofoil with a beveled trailing edge. Model. Simul. Eng. 2015, 565417. [CrossRef]

64. Lee, S.-J. Numerical Simulation of Vortex-Dominated Flows Using the Penalized VIC Method. In Vortex Dynamics and Optical Vortices; Perez-de-Tejada, H., Ed.; InTech: London, UK, 2017. [CrossRef]

65. Baker, A.H.; Falgout, R.D.; Kolev, T.V.; Yang, U.M. Scaling hypre's Multigrid Solvers to 100,000 Cores. In High Performance Scientific Computing: Algorithms and Applications; Berry, M.W., Gallivan, K.A., Gallopoulos, E., Grama, A., Philippe, B., Saad, Y., Saied, F., Eds.; Springer: Berlin/Heidelberg, Germany, 2012.

66. Koumoutsakos, P.; Leonard, A. High-resolution simulations of the flow around an impulsively started cylinder using vortex methods. J. Fluid Mech. 1995, 296, 1-38. [CrossRef]

67. Bouard, R.; Coutanceau, M. The early stage of development of the wake behind an impulsively started cylinder for $40<R e<104$. J. Fluid Mech. 1980, 101, 583-607. [CrossRef]

68. Miller, L.A.; Peskin, C.S. When vortices stick: An aerodynamic transition in tiny insect flight. J. Exp. Biol. 2004, 207, 3073-3088. [CrossRef]

69. Kerho, M.; Kramer, B.R. Research Water Tunnels—Specification; Rolling Hills Research Corporation (RHRC): El Segundo, CA, USA, 2003. 